\title{
Epigenetic modifications and gestational diabetes: a systematic review of published literature
}

\author{
Gunn-Helen Moen ${ }^{1,2}$, Christine Sommer ${ }^{1}$, Rashmi B Prasad $^{3}$, Line Sletner, \\ Leif Groop $^{3,6}$, Elisabeth Qvigstad ${ }^{1}$ and Kåre I Birkeland 1,2,7 \\ 'Department of Endocrinology, Morbid Obesity and Preventive Medicine, Oslo University Hospital, \\ Oslo, Norway, ${ }^{2}$ Faculty of Medicine, University of Oslo, Institute of Clinical Medicine, Oslo, Norway, \\ ${ }^{3}$ Department of Clinical Sciences, Diabetes and Endocrinology CRC, Lund University Diabetes Centre, \\ Malmö, Sweden, ${ }^{4}$ Department of Pediatric and Adolescents Medicine, Akershus University Hospital, \\ Lørenskog, Norway, ${ }^{5}$ MRC Lifecourse Epidemiology Unit, University of Southampton, \\ Southampton General Hospital, Southampton, UK, ${ }^{6}$ Finnish Institute of Molecular Medicine (FIMM), \\ Helsinki University, Helsinki, Finland, and ${ }^{7}$ Department of Transplantation Medicine, Oslo University \\ Hospital, Oslo, Norway
}

Correspondence should be addressed to G-H Moen

Email

g.h.o.moen@studmed.uio.no

\begin{abstract}
Objective: To summarize the current knowledge on epigenetic alterations in mother and offspring subjected to gestational diabetes (GDM) and indicate future topics for research.

Design: Systematic review.

Methods: We performed extensive searches in PubMed, EMBASE and Google scholar, using a combination of the search terms: GDM, gestational diabetes, epigenetic(s), methylation, histone modification, histone methylation, histone acetylation, microRNA and miRNA. Studies that compared women diagnosed with GDM and healthy controls were included. Two authors independently scanned the abstracts, and all included papers were read by at least two authors. The searches were completed on October 31st, 2016.

Results: We identified 236 articles, of which 43 were considered relevant for this systematic review. Studies published showed that epigenetic alterations could be found in both mothers with GDM and their offspring. However, differences in methodology, diagnostic criteria for GDM and populations studied, together with a limited number of published studies and small sample sizes, preclude clear conclusions about the role of epigenetic modifications in transmitting risk from GDM mothers to their offspring.

Conclusion: The current research literature suggests that GDM may have impact on epigenetic modifications in the mother and offspring. However, larger studies that include multiple cohorts of GDM patients and their offspring are needed.
\end{abstract}

\section{Invited Author's profile}

Gunn-Helen Moen is currently studying for a PhD at the University of Oslo Faculty of Medicine/Oslo University hospital, Department of Endocrinology, Morbid Obesity and Preventive Medicine. She is a member of the Oslo Diabetes Research Center and is associated with two research groups: one investigating diabetes in pregnancy and one focusing on type 2 diabetes and metabolism. Her research focuses on genetic and epigenetic aspects of gestational diabetes and the consequences of hyperglycemia in pregnancy for both mother and child.

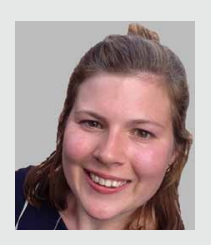

() 2017 European Society of Endocrinology Printed in Great Britain
Published by Bioscientifica Ltd.
European Journal of Endocrinology

(2017) 176, R247-R267 


\section{Introduction}

Gestational diabetes (GDM) defined as glucose intolerance first recognized in pregnancy, may have important implications for both mother and fetus. Offspring of mothers with GDM have an increased risk of birth complications associated with increased birth weight and adiposity, such as shoulder dystocia, neonatal hypoglycemia and obesity, as well as for developing metabolic syndrome, type 2 diabetes (T2DM) and cardiovascular disease in adult life $(1,2)$. Furthermore, in a 5- to 10-year perspective, it is estimated that around $50 \%$ of the women diagnosed with GDM will develop T2DM, although the subsequent T2DM risk varies greatly with criteria used and follow-up time $(3,4)$. As GDM and T2DM appear to share both genetic and non-genetic risk factors, the overlapping susceptibility may partly explain the increased risk of T2DM for women with previous $\operatorname{GDM}(5,6)$.

The Forsdahl-Barker hypothesis suggested that maternal undernutrition and low-birth-weight was associated with chronic disease, such as T2DM, in adult life $(7,8,9,10,11,12,13)$. However, in populations where T2DM is prevalent, the relationship between birth weight and later T2DM has been reported to be U-shaped (14). Some of the reported risk alleles known to influence susceptibility of T2DM support such a U-shaped relationship between birth weight and later T2DM, as some genes show association with lower birth weight and some with higher birth weight (15). Moreover, the fetal insulin hypothesis suggests that the observed association between low-birth-weight and adult insulin resistance is mediated through genetics $(16,17,18)$, indicating that low fetal growth is a sign of genetically determined insulin resistance leading to glucose intolerance later in life. In addition, the current obesity epidemic raises concerns that overnutrition in utero can lead to permanent metabolic changes in the fetus and increase the risk of adiposity and diabetes in later life (19).

Today, more than 100 different SNPs have been reported to associate with T2DM (20). However, these candidate genes only explain a small proportion of the heritability, and environmental influence is obviously important in the pathogenesis. The evidence for a genetic predisposition for GDM is not as robust as that for T2DM (5), but several systematic reviews and meta-analyses have highlighted genes such as Transcription Factor 7 Like 2 (TCF7L2), Melatonin receptor 1B (MTNR1B) and Insulin receptor substrate 1 (IRS1) among others to be important $(21,22,23,24,25,26)$. Epigenetic modifications during pregnancy could be an interesting method to study the environmental influence (27), as several studies have reported the effect of GDM on birth outcomes and indicated that epigenetic alterations may be an important mediator (28). One example is the increased risk of obesity and T2DM in siblings born after the mother developed diabetes compared to siblings born before the mother was diagnosed (29). Studies have also shown that GDM is more common in daughters of diabetic mothers compared to that of diabetic fathers $(30,31)$, suggesting that the intrauterine environment, in addition to genetics, is important. In addition, it has been shown that parental genotypes, even when these genes are not inherited by the offspring, can lead to epigenetic alterations in the offspring, which in turn may affect the offspring's phenotype $(32,33,34)$.

Epigenetics refer to coding, excluding that of the DNA sequence, which can influence the transcriptional rate. These variations in the epigenetic programming can be caused by environmental factors and can activate or silence gene expression. The type of modification can vary, but the most common ones are DNA methylation, modification of histones or regulation of gene expression by microRNA (miRNA).

Methylation of cytosine on CpG sites in the DNA, creating methylcytosine $(5-\mathrm{mC})$, is the most frequently studied modification. Methylation can silence repressor elements and lead to increased gene expression, whereas methylation at the promoter or enhancer regions can decrease gene expression, the latter by preventing enhancer elements access to the region. It has also been shown that methylation in the gene body can increase the expression or influence alternative splicing (35). Addition of a hydroxyl group to an already methylated cytosine, creating hydroxymethylcytosine (5-hmC), is another epigenetic regulatory marker, suggested to promote DNA demethylation (36). Epigenetic regulation via histone modification can influence the chromatin packing and subsequently the gene expression. For instance, methylation at histone 3 , lysine 4 (H3K4) is known to activate genes, whereas methylation of histone 3 lysine 27 (H3K27) is generally associated with gene repression (37). Finally, miRNA, short non-coding RNAs, is involved in post-transcriptional regulation of gene expression. miRNA can affect both the stability and translation of mRNA. Most often miRNA binding to mRNA leads to translational inhibition or destabilization (38).

The aim of this systematic review is to summarize the current knowledge on epigenetic alterations in mother 
and offspring subjected to gestational diabetes and indicate future topics for research.

\section{Methods}

This systematic review was conducted in agreement with the PRISMA statement for reporting systematic reviews (39). The protocol for this systematic review was registered in the international prospective register of systematic reviews (PROSPERO) (http://www.crd.york.ac.uk/ PROSPERO, registration number is: CRD42016036103).

We performed extensive searches in PubMed, EMBASE and Google scholar, using a combination of the search terms: GDM, gestational diabetes, epigenetic(s), methylation, histone modification, histone methylation, histone acetylation, microRNA and miRNA. The initial searches were continuously updated from March 1st 2016 to October 31st 2016, and no publication date or publication restrictions were imposed. Scanning of search results and identification of relevant articles according to inclusion and exclusion criteria were performed separately by two reviewers (GHM and CS). All articles were read by at least two independent reviewers (GHM, EQ and KIB).

We included all articles that studied gestational diabetes in association with epigenetic modificationssuch as DNA methylation, histone modifications or miRNA change-in tissues from the mother or her offspring. We included studies using consensus-based criteria based on an oral glucose tolerance test (OGTT). The different criteria are described in Table 1. If diagnostic criteria were not reported in detail, the authors were asked to provide this information. We excluded animal studies and articles studying pre-gestational diabetes. We also excluded studies with less than 10 GDM cases in total; however, a short summary of these five studies is added in the Results section. Only papers in English were considered eligible, but no relevant studies were excluded due to language.
From each study, data on (1) maternal blood glucose, (2) method, (3) methylation status and/or status on histone modifications and miRNA expression, (4) diagnosis criteria and (5) ethnicity were extracted. In articles where diagnostic criteria were not reported, some authors provided this information upon request. The primary outcome looked at in this review were methylation changes as a result of GDM, either as correlation between blood glucose and methylation status or as mean differences in methylation between GDM pregnancies and healthy controls. A descriptive presentation of results was chosen, and formal meta-analyses were not performed due to considerable heterogeneity between studies.

To ascertain the quality of the eligible studies, the reviewers worked independently to determine if relevant diagnostic criteria for GDM were used, as well as establish that the GDM and control groups were properly matched. In addition, the reviewers assessed if proper statistical analysis was performed. Lastly, risk of publication bias and selective reporting within each study were assessed.

\section{The current evidence}

Of 236 articles retrieved, 38 articles were considered relevant for this systematic review (Fig. 1). Of these, 12 were genome-wide methylation studies, 4 used broad array approaches on miRNA and 22 reported epigenetic modifications in candidate genes or specific miRNAs. Placental tissue was used in 23 studies and umbilical cord blood (UCB) in 12 studies (whereof 7 studied both placental tissue and umbilical cord blood). Maternal peripheral blood or fat tissue was used in five studies and peripheral blood or muscle and fat tissue from the offspring in two studies. Human umbilical vein endothelial cells (HUVECs) were used in three studies. The majority reported DNA methylation; two studies reported histone modifications and eight studies reported changes in miRNA. The main findings, study population and population size of each study can be found in the Tables 2, 3 and 4. A summary of findings in the placenta are visualized in Figure 2.

Table 1 Diagnostic criteria.

\begin{tabular}{|c|c|c|c|c|c|}
\hline & Glucose load & Fasting value (mmol/L) & 1-h value $(\mathrm{mmol} / \mathrm{L})$ & 2-h value $(\mathrm{mmol} / \mathrm{L})$ & 3-h value $(\mathrm{mmol} / \mathrm{L})$ \\
\hline WHO 1999 & $75 g$ & $\geq 7.0$ & - & $\geq 7.8$ & - \\
\hline ADA $2003 /$ Carpenter and Coustan & $75 \mathrm{~g} / 100 \mathrm{~g}$ & $\geq 5.3$ & $\geq 10.0$ & $\geq 8.6$ & $\begin{array}{l}\geq 7.8 \text { (only with } \\
100 \mathrm{~g} \text { glucose) }\end{array}$ \\
\hline $\begin{array}{l}\text { IADPSG 2010/WHO 2013/ADA 2013/ } \\
\text { GDA/Chinese MOH } 2011\end{array}$ & $75 \mathrm{~g}$ & $\geq 5.1$ & $\geq 10.0$ & $\geq 8.5$ & - \\
\hline National Diabetes Data Group & $100 \mathrm{~g}$ & $\geq 5.8$ & $\geq 10.6$ & $\geq 9.2$ & $\geq 8.1$ \\
\hline
\end{tabular}

ADA, American Diabetes Association; GDA, German Diabetes Association; IADPSG, International Association of the Diabetes and Pregnancy Study Groups; $\mathrm{MOH}$, Ministry of Health; WHO, World Health Organization. 


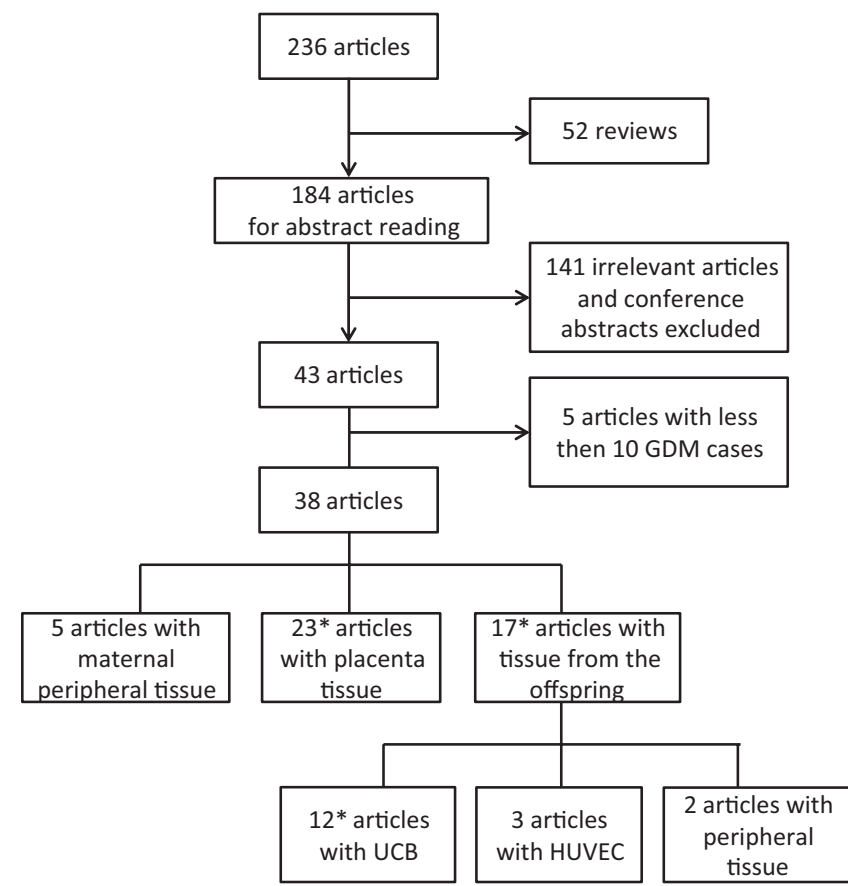

Figure 1

Flow of information through the systematic review. *7 studies on both placenta and UCB. HUVEC, human umbilical vein endothelial cells; UCB, umbilical cord blood.

\section{Epigenetic alterations in the mother}

\section{Maternal peripheral blood}

A study in peripheral white blood cells (WBC) of 22 women (40) examined the global methylation pattern sampled in gestational week (GW) 12-16 and identified five CpG sites that were potentially differentially methylated. However, the results were not significant when adjusted for false discovery rate (FDR). A technical validation between array $\beta$-values and methylation levels was determined by bisulfite pyrosequencing (BSP). The authors report that by the use of these differentially methylated genes, they could predict the development of GDM in at least eight of the eleven women. However, they did not adjust their prediction model for ambient glucose or HbA1c levels, even though some women who develop GDM may have slightly elevated blood glucose already at 12-16 weeks.

A case-control study in maternal plasma from 20 women (41) showed that 32 miRNA was differently expressed in GDM women compared to controls in GW 16-19. Targets of these miRNAs were, among others, associated with insulin signaling and T2DM. The authors validated the altered expression in five miRNAs.
A study in maternal serum from 48 women (42) showed different levels of miR-132, miR-29a and miR222 between pooled samples from women who later developed GDM and controls in GW 16-19. The results were validated internally and miR-29a and miR-222 were also externally validated. The study further demonstrated that knockdown of miR-29a, in HepG2 cell line, could increase insulin-induced gene 1 (INSIG1) gene expression, involved in glucose homeostasis. The authors could not identify a clear biological function of miR-132 and miR222 related to GDM.

A study in 27 multi-ethnic women (43) reported on global histone $\mathrm{H} 3$ dimethylation as an epigenetic marker for prediction of conversion from GDM to T2DM. H3K27 and H3K4 dimethylation was lower in women with GDM who later developed T2DM than that in GDM women who did not. However, the groups were small (6-8 in each groups) and heterogeneous regarding ethnicity and parity, which may bias the results. Furthermore, the follow-up time after pregnancy was short (a total follow-up time of eight months), and some GDM women could develop T2DM later.

\section{Maternal adipose tissue}

A study in omental adipose tissue taken at the time of Cesarean section from 26 women (44) identified 17 differentially expressed miRNA between GDM women and controls. Samples from three GDM women and three controls were run on a miRNA expression chip, and expression of miR-222 was validated by q-PCR in all 13 cases and 13 controls. miR-222 expression was negatively correlated with estrogen receptor (ER) $\alpha$ and glucose transporter 4 (GLUT4) protein levels. Furthermore, serum levels of estradiol correlated with miR-222 expression, and the authors suggested that miR-222 may be involved in estrogen-induced insulin resistance via ER $\alpha$ and GLUT4.

\section{Epigenetic alterations in the placenta}

A total of 23 articles on epigenetic changes in placenta samples were included, 16 of which only reported findings in placenta and seven of which reported findings both in placenta and umbilical cord blood. The three articles with key data in both placenta and umbilical cord tissue are discussed separately in this section and the next looking at epigenetic alteration in the offspring. The five with main results from only one tissue is discussed in the appropriate section and in Table 3 or 4 . Not all the studies reported which side of the placenta the samples were taken from, 
Table 2 Main characteristics of the studies included using maternal peripheral blood.

\begin{tabular}{|c|c|c|c|c|c|c|c|c|}
\hline Reference & Study design & Method & Tissue & GDM criteria & Main finding & $n$ & Statistics & Comment \\
\hline \multirow[t]{2}{*}{ (40) } & $\overline{\text { Case-control }}$ & $\begin{array}{l}\text { Genome-wide } \\
\text { DNA } \\
\text { methylation } \\
(450 \mathrm{~K})\end{array}$ & $\begin{array}{l}\text { Maternal } \\
\text { WBC }\end{array}$ & $\begin{array}{l}\text { Fasting PG: } \\
\geq 5.6 \mathrm{mmol} / \mathrm{L}\end{array}$ & $\begin{array}{l}8 \text { of } 11 \text { women } \\
\text { who } \\
\text { developed } \\
\text { GDM showed } \\
\text { differential } \\
\text { methylation } \\
\text { at } 5 \text { CpGs }\end{array}$ & $\overline{22(11 \mathrm{GDM})}$ & $\begin{array}{l}\text { Cut of B-value } \\
\text { difference of } \\
\geq 0.2 \\
\text { between } \\
\text { cases and } \\
\text { controls }\end{array}$ & $\begin{array}{l}\text { EFFECT-M } \\
\text { study }\end{array}$ \\
\hline & & & & $\begin{array}{l}\text { 2-h PG: } \\
\geq 7.8 \mathrm{mmol} / \mathrm{L}\end{array}$ & & & $\begin{array}{l}\text { Not significant } \\
\text { after FDR } \\
\text { adjustment }\end{array}$ & \\
\hline \multirow[t]{2}{*}{ (41) } & Case-control & $\begin{array}{l}\text { High- } \\
\text { throughput } \\
\text { sequencing } \\
\text { (lon Torrent) } \\
\text { and qRT-PCR }\end{array}$ & $\begin{array}{l}\text { Maternal } \\
\text { plasma }\end{array}$ & ADA 2003 & $\begin{array}{l}32 \text { miRNA were } \\
\text { differently } \\
\text { expressed }\end{array}$ & 20 (10 GDM) & $\begin{array}{l}\text { Significance } \\
\text { level: } P \leq 0.05\end{array}$ & $\begin{array}{l}\text { Pooled } \\
\text { samples }\end{array}$ \\
\hline & & & & & & & & $\begin{array}{l}\text { Sampled at } \\
\text { Zhongda } \\
\text { Hospital, } \\
\text { Nanjing, } \\
\text { China }\end{array}$ \\
\hline$(42)$ & Case-control & $\begin{array}{l}\text { TaqMan low } \\
\text { density array } \\
\text { and qRT-PCR }\end{array}$ & $\begin{array}{l}\text { Maternal } \\
\text { serum }\end{array}$ & ADA 2003 & $\begin{array}{l}\text { Different levels } \\
\text { of miR-132, } \\
\text { miR-29a and } \\
\text { miR-222 }\end{array}$ & 48 (24 GDM) & $\begin{array}{l}\text { Significance } \\
\text { level: } P \leq 0.05\end{array}$ & $\begin{array}{l}\text { Pooled } \\
\text { samples }\end{array}$ \\
\hline (43) & Cohort & $\begin{array}{l}\text { Histone } \\
\text { methylation } \\
\text { at five lysine } \\
\text { sites }\end{array}$ & $\begin{array}{l}\text { Maternal } \\
\text { WBC }\end{array}$ & IADPSG 2010 & $\begin{array}{l}75 \% \text { reduction } \\
\text { in H3K4 } \\
\text { dimethylation } \\
8-10 \text { weeks } \\
\text { postpartum in } \\
\text { women who } \\
\text { had GDM and } \\
\text { developed } \\
\text { T2DM and } \\
\text { GDM women } \\
\text { that did not }\end{array}$ & $\begin{array}{l}39 \text { (6-8 in } \\
\text { each of } \\
\text { the four } \\
\text { groups) }\end{array}$ & $\begin{array}{l}\text { Did not } \\
\text { correct for } \\
\text { multiple } \\
\text { testing, } \\
P \leq 0.05\end{array}$ & $\begin{array}{l}\text { Difference } \\
\text { between } \\
\text { the groups } \\
\text { regarding } \\
\text { ethnic } \\
\text { distribution }\end{array}$ \\
\hline (44) & Case-control & $\begin{array}{l}\text { AFFX miRNA } \\
\text { expression } \\
\text { chip and } \\
\text { qRT-PCR }\end{array}$ & $\begin{array}{l}\text { Maternal } \\
\text { Omental } \\
\text { adipose } \\
\text { tissue }\end{array}$ & ADA 2003 & $\begin{array}{l}\text { miR-222 was } \\
\text { differently } \\
\text { expressed and } \\
\text { negatively } \\
\text { correlated } \\
\text { with ER } \alpha \\
\text { protein levels }\end{array}$ & 26 (13 GDM) & $\begin{array}{l}\text { miRNA fold } \\
\text { change of at } \\
\text { least } 1.5 \\
\text { between } \\
\text { GDM and } \\
\text { controls }\end{array}$ & $\begin{array}{l}3 \text { GDM vs } 3 \\
\text { controls on } \\
\text { miRNA } \\
\text { chip. } 10 \\
\text { cases and } \\
\text { controls in } \\
\text { validation }\end{array}$ \\
\hline & & & & & & & $\begin{array}{l}\text { Significance } \\
\text { level: } P \leq 0.05\end{array}$ & $\begin{array}{l}\text { Samples } \\
\text { obtained in } \\
\text { Nanjing } \\
\text { Medical } \\
\text { University, } \\
\text { China }\end{array}$ \\
\hline
\end{tabular}

ADA, American Diabetes Association; ER $\alpha$, estrogen receptor $\alpha$; FDR, false discovery rate; GDM, gestational diabetes; IADPSG, International Association of the Diabetes and Pregnancy Study Groups; miRNA, microRNA; PG, plasma glucose; qRT-PCR, real-time polymerase chain reaction; T2DM,

type 2 diabetes; WBC, white blood cells.

so we have chosen to report all findings from the placenta here. The results are summarized in Table 3.

\section{LEP}

A study of 535 healthy term pregnancies in a multi-ethnic cohort (45) showed a $2.5 \%$ higher degree of methylation of the leptin $(L E P)$ gene on the fetal side of the placenta of 47 women with GDM. Further, a study on 48 Canadian women (46) found a significant correlation between the 2-h glucose value and the degree of DNA methylation of the LEP gene in placenta on both fetal and maternal side in 23 GDM women. Higher glucose values correlated with lower degree of methylation on the fetal side, but with a 


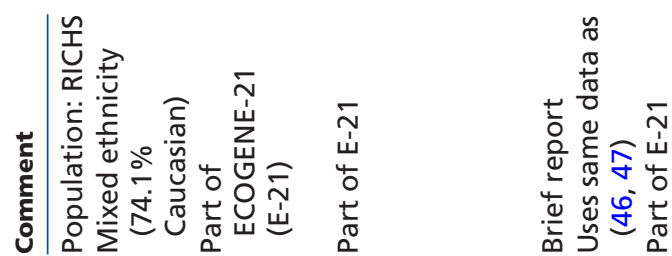

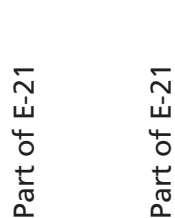
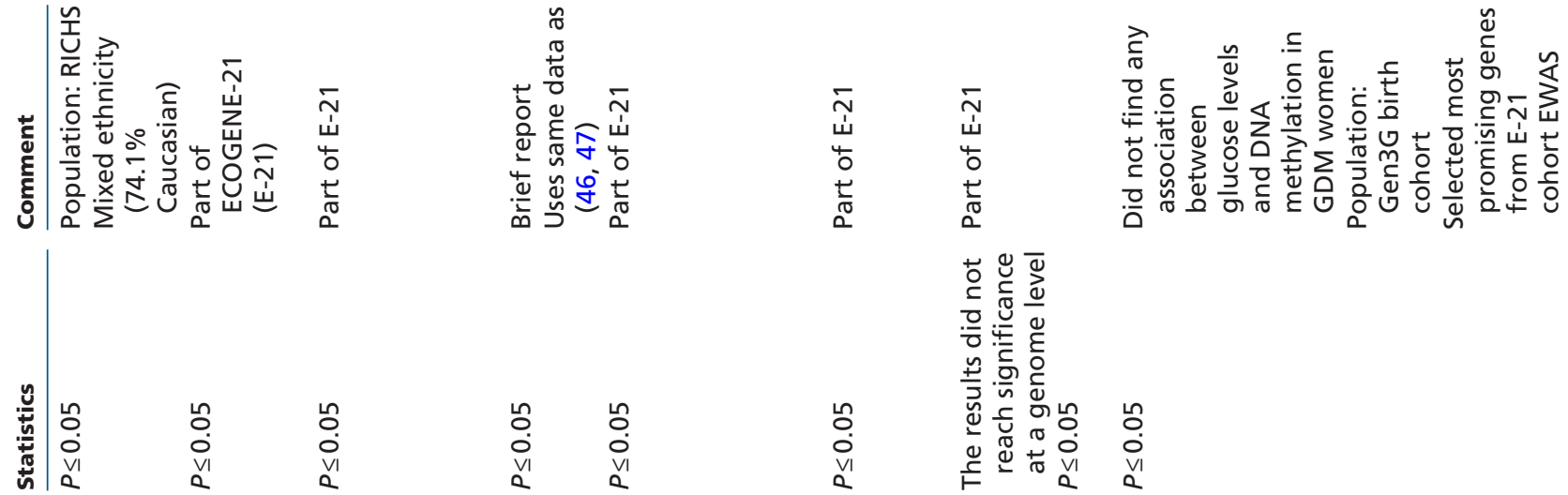

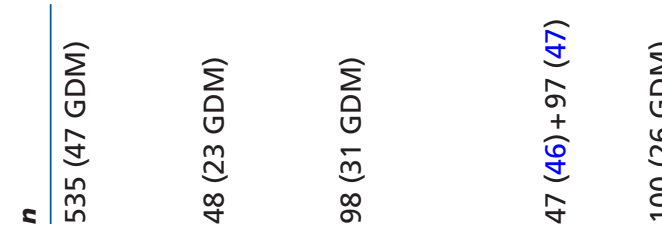

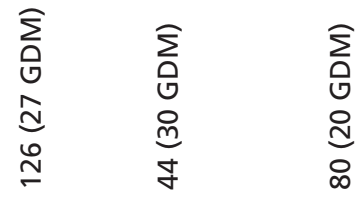

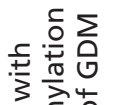

(

-
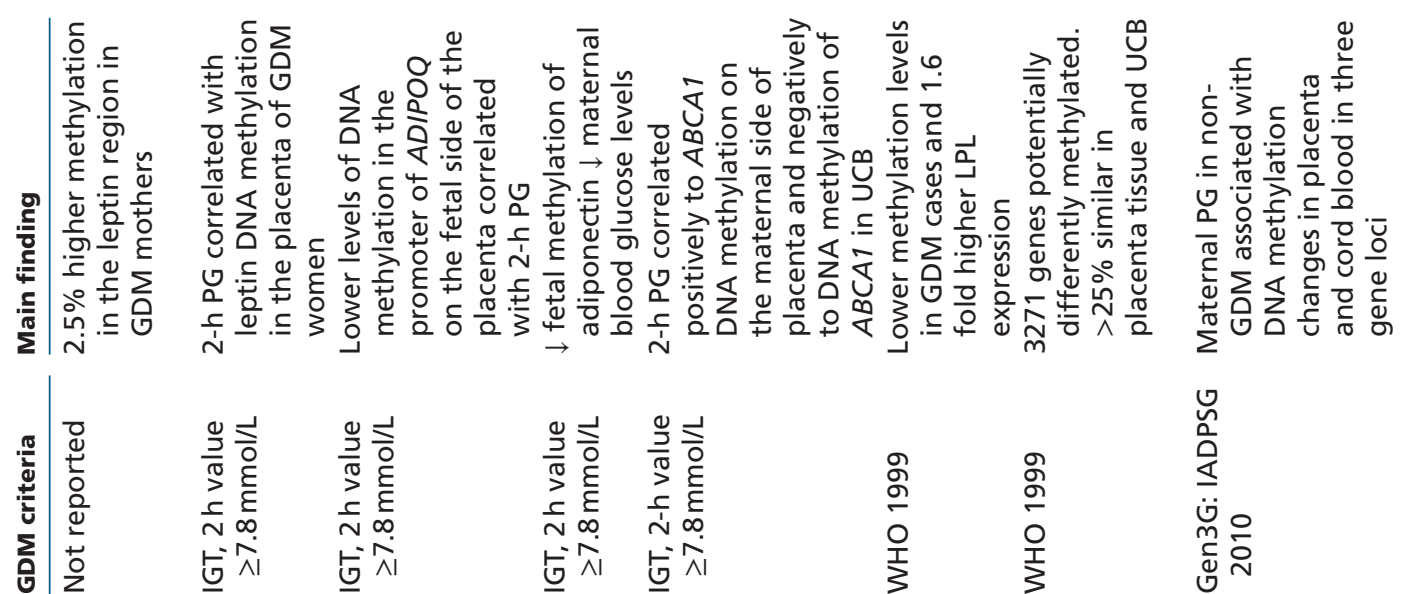

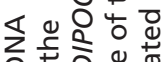

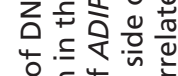

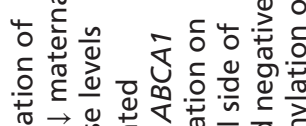

두을

$\frac{\pi}{\lambda}$

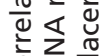

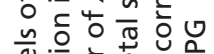

ते.

这势

等.

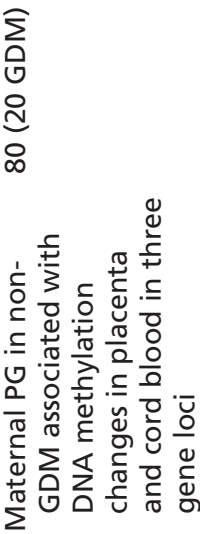
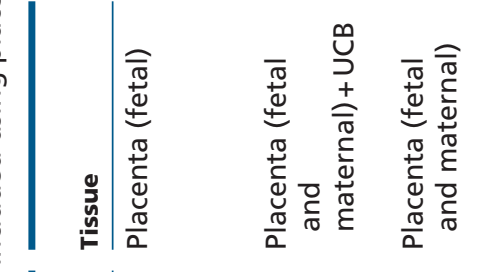

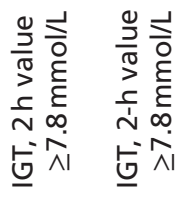

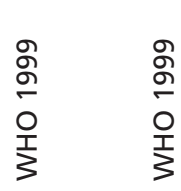

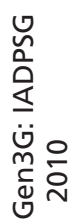

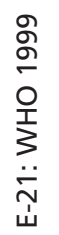

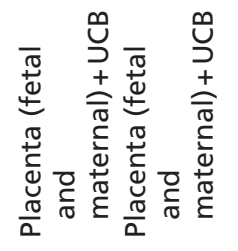

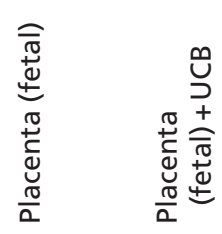

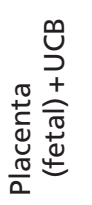

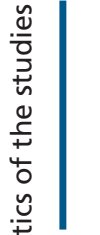

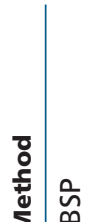

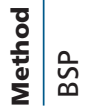

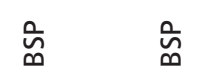

ڤิ

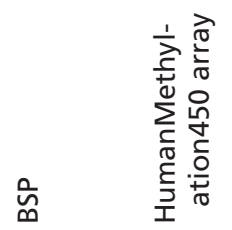

ติ

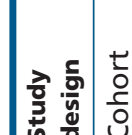

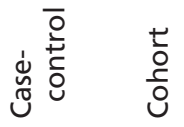

$\begin{array}{ll}\frac{\pi}{0} & \frac{t}{0} \\ \frac{0}{0} & \frac{c}{0}\end{array}$

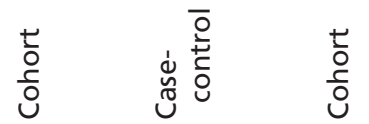

$\stackrel{\frac{5}{\pi}}{\Sigma}$

$\frac{1}{\pi}$
$m$
$\frac{0}{0}$
$\frac{0}{10}$

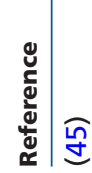

孚压

票 襄

宫店

$\widehat{\widetilde{G}}$ 


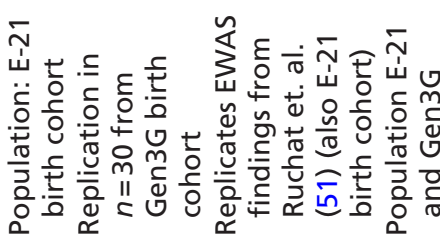

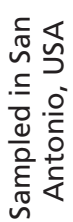

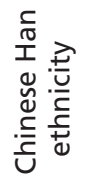

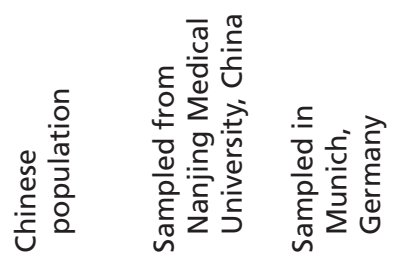
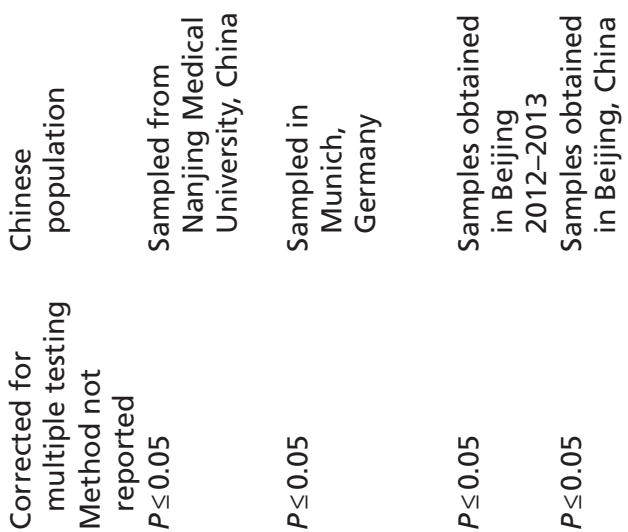

Ln
$\dot{0}$
Vi
$\alpha$

$\begin{array}{ll}n & n \\ 0 & 0 \\ 0 & 0 \\ \text { vil } & \text { v } \\ a & a\end{array}$

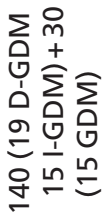

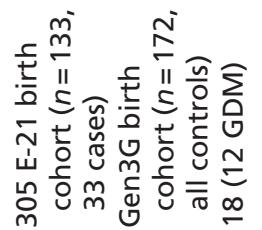

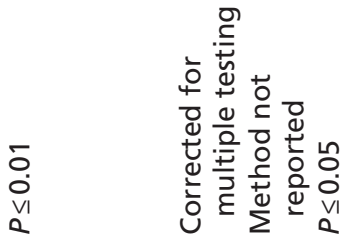

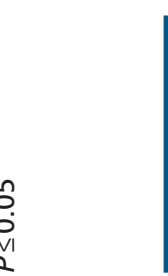

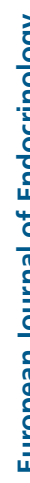

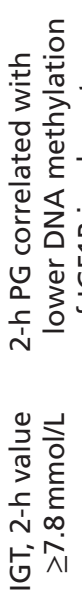

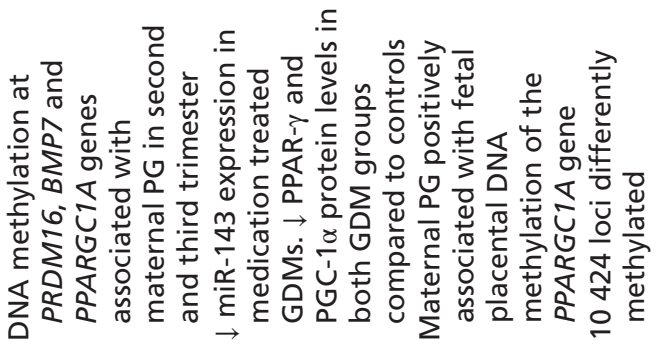

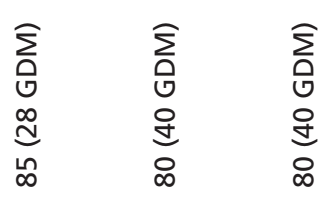

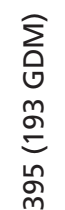

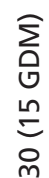

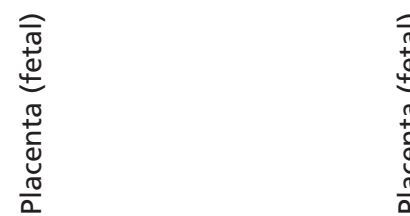

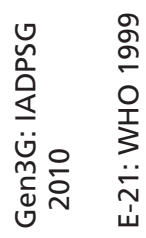

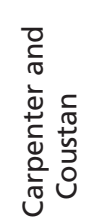

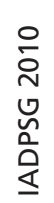

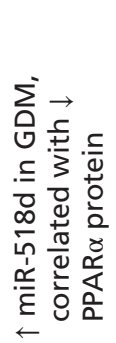

造

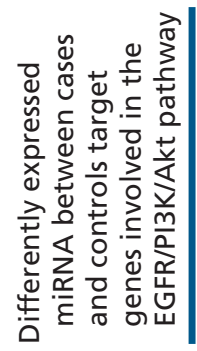

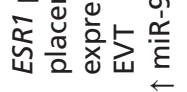
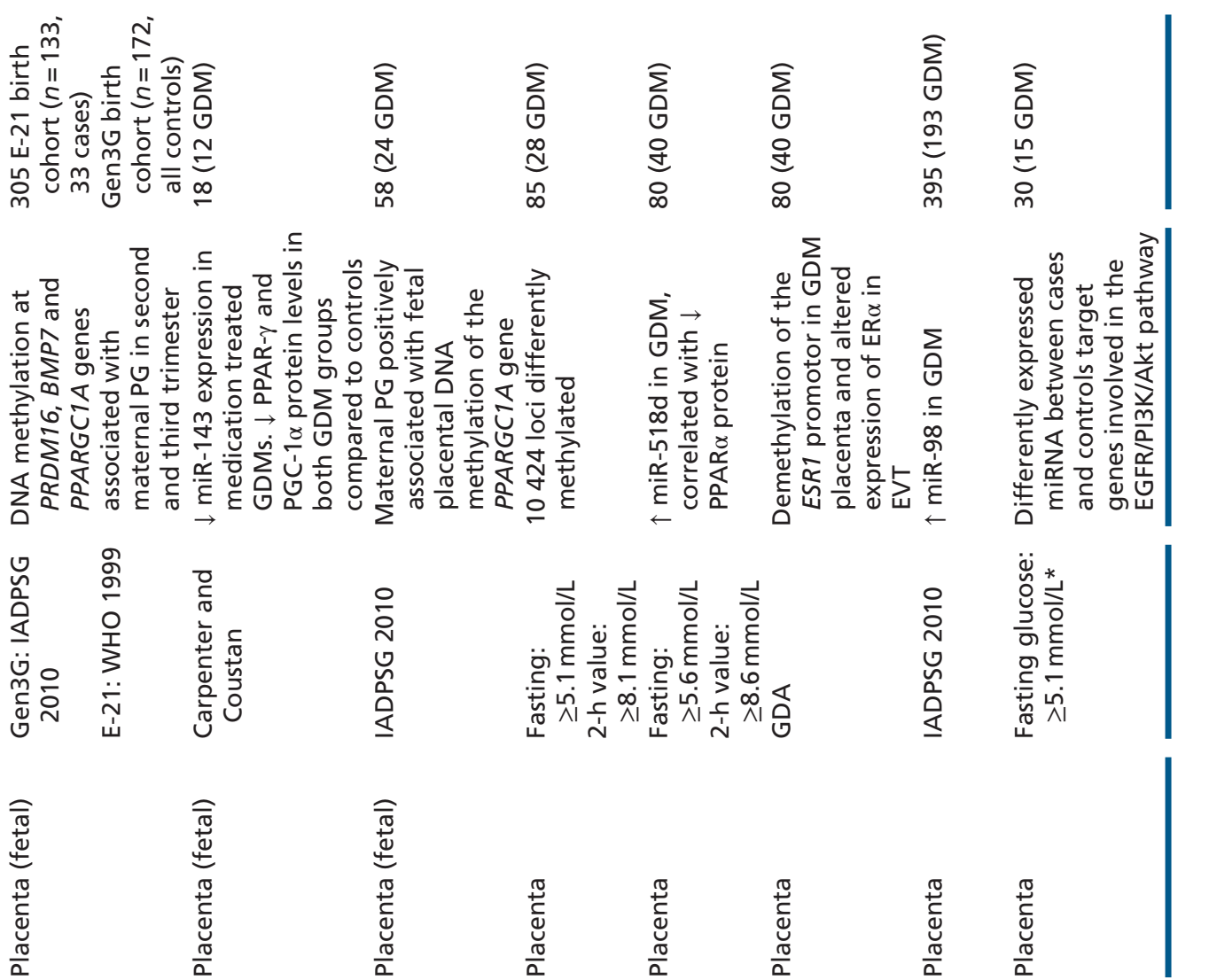

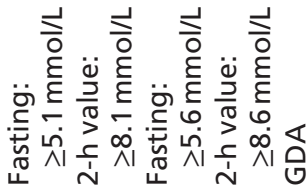

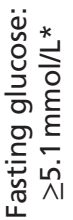
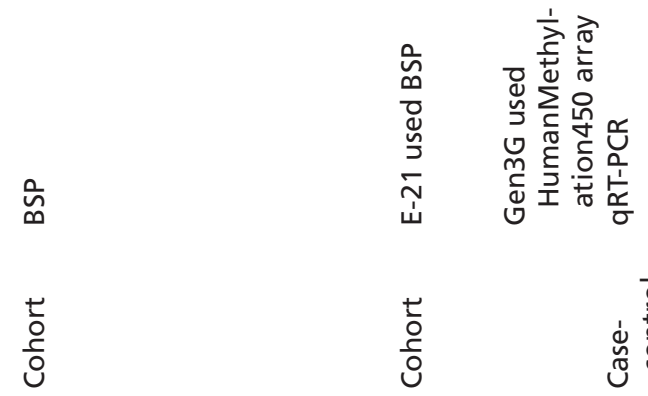

$\begin{array}{ll}\frac{\pi}{\pi} & \frac{\pi}{0} \\ \stackrel{0}{0} & \frac{\pi}{0} \\ \frac{\pi}{0} & \frac{\pi}{0}\end{array}$

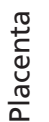

$\begin{array}{ll}\stackrel{\pi}{0} & \stackrel{0}{0} \\ \stackrel{0}{0} & \frac{0}{4} \\ \frac{\pi}{0} & \frac{\pi}{0}\end{array}$

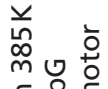

ه

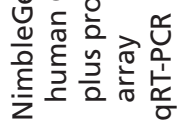

高

ถิ

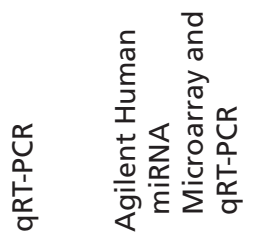

$\frac{t}{0}$
$\frac{0}{0}$
O

نे

نั

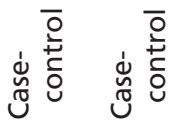

苞

否

葛

啇

ह6

畐

畣

ब요 


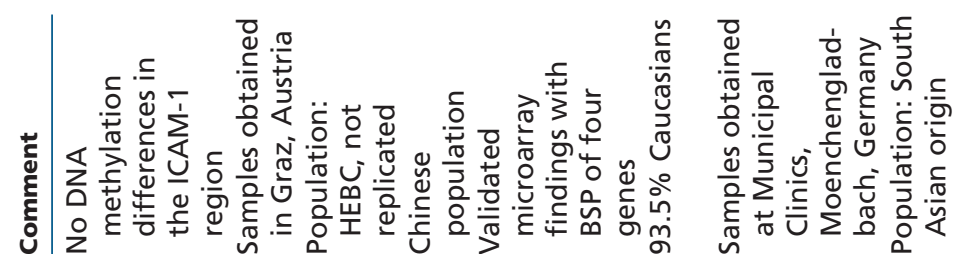

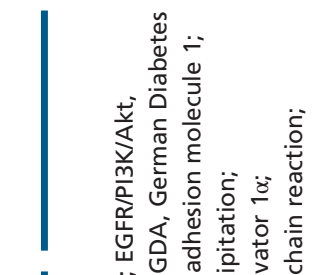

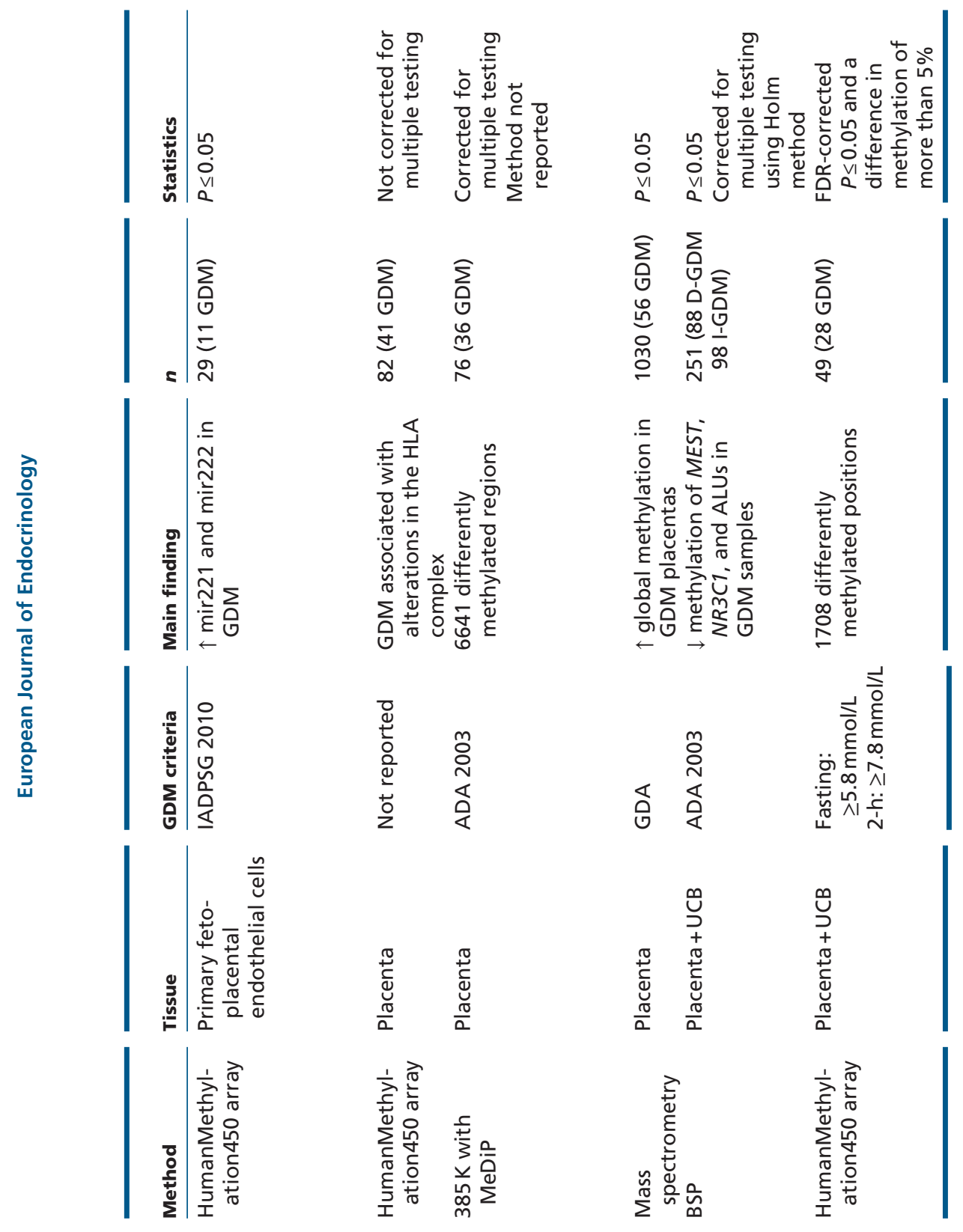

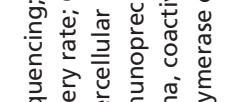

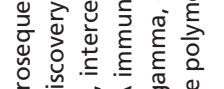

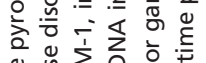

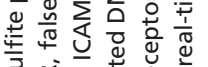

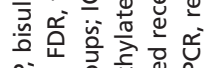

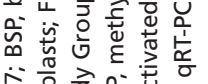

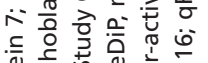

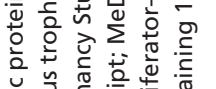

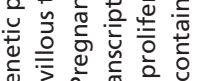

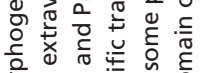

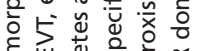

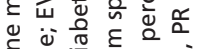

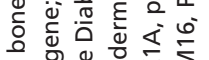

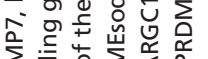

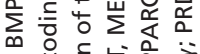

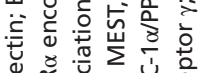

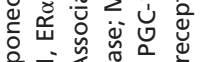

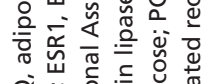

वั

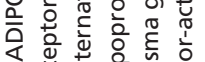

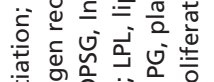

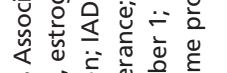

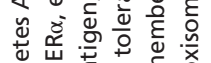

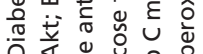

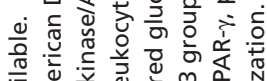

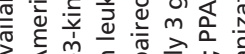

कर

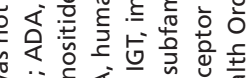

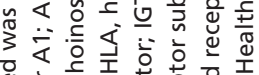

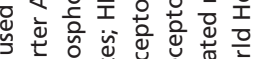

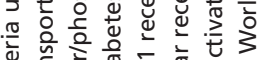

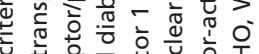

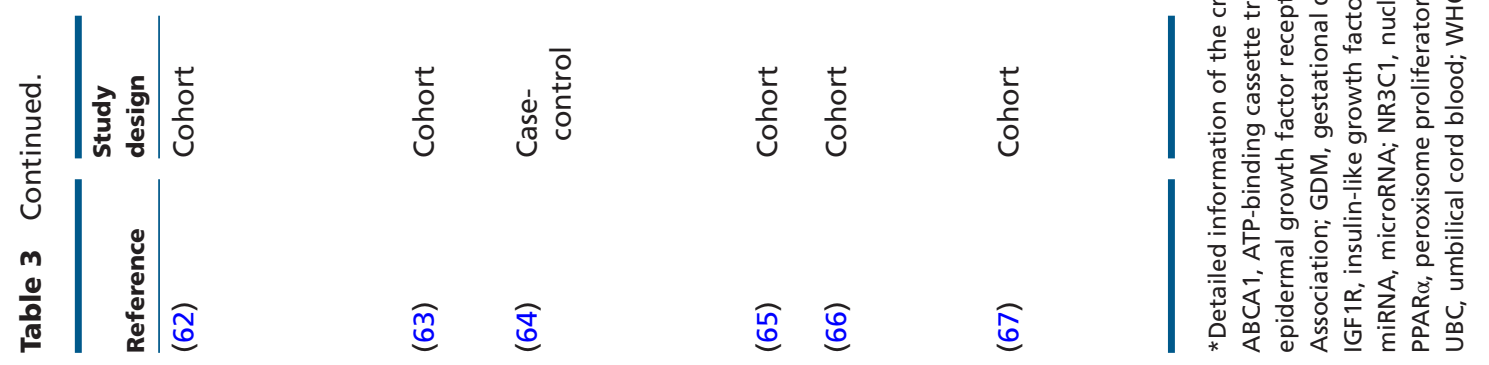




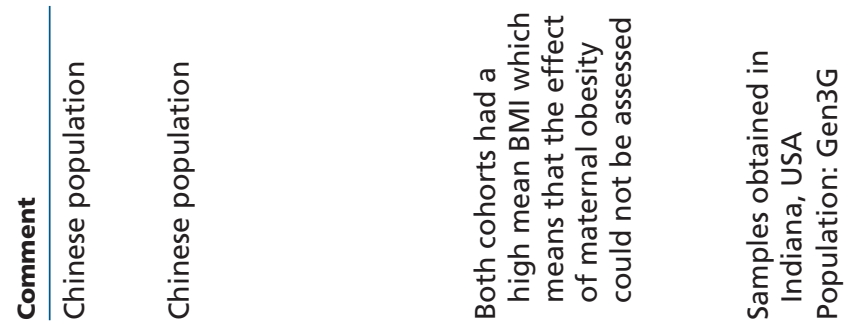

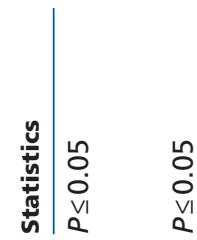

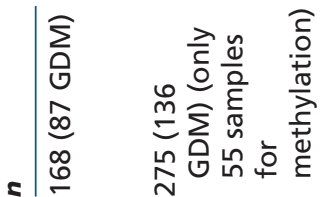

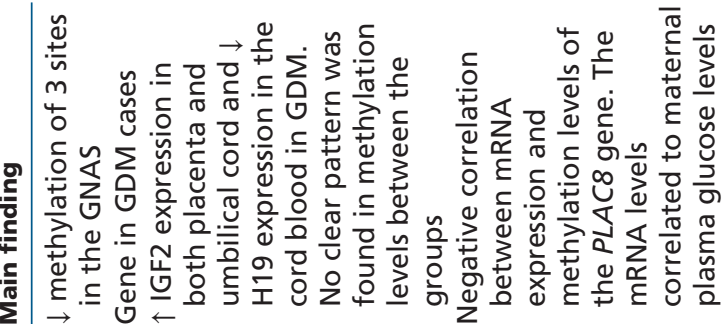

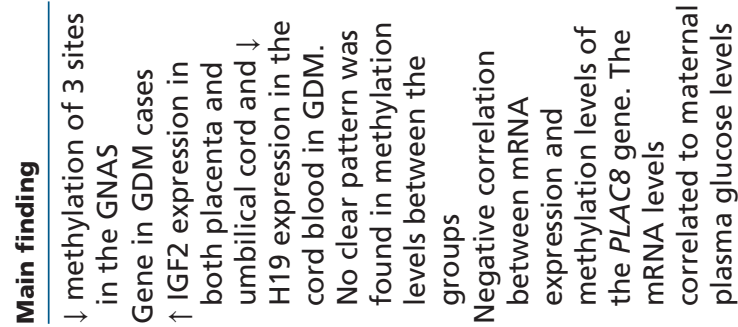

.

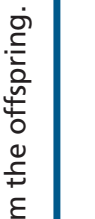

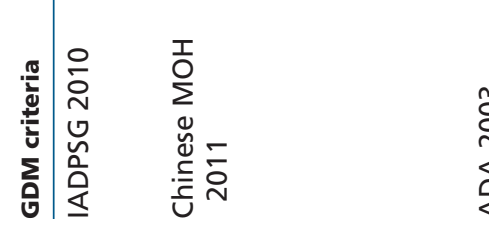

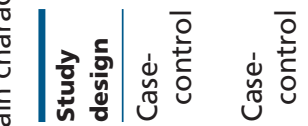

$\sum$

$\frac{\pi}{0}$
$\frac{0}{0}$

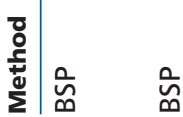

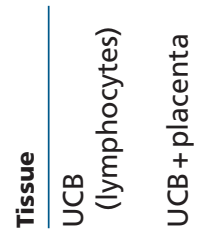

ญ̊

ڤิ

空

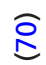

L
0
0
vin
0

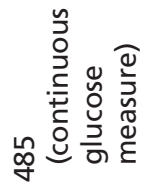

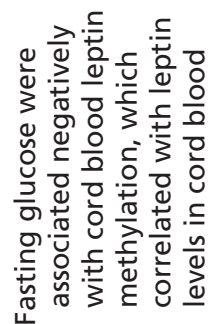

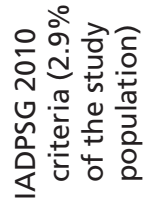

ญั

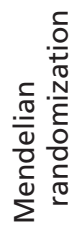

능

ล
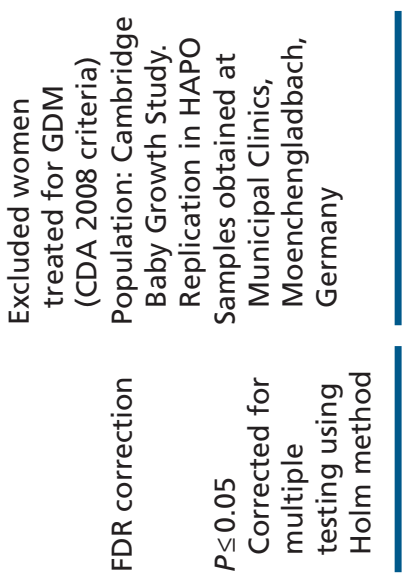

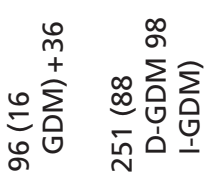

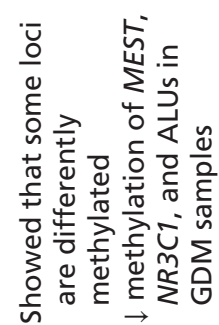

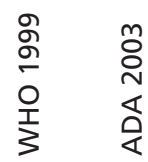
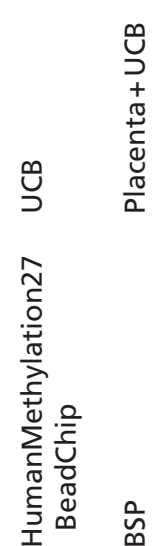

言

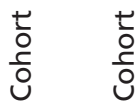

న 

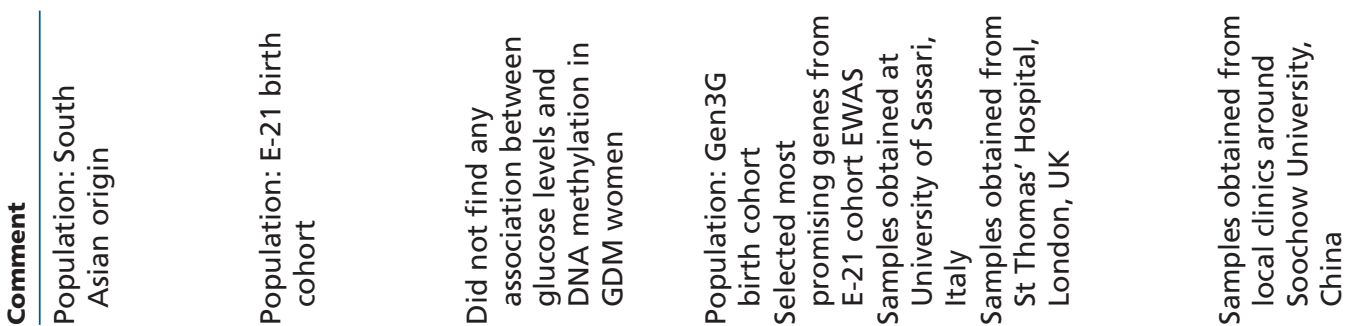

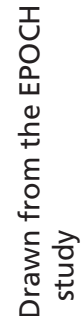

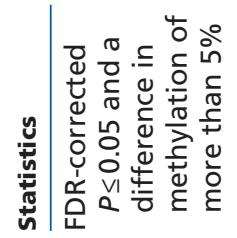

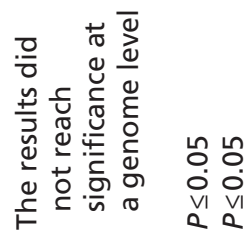
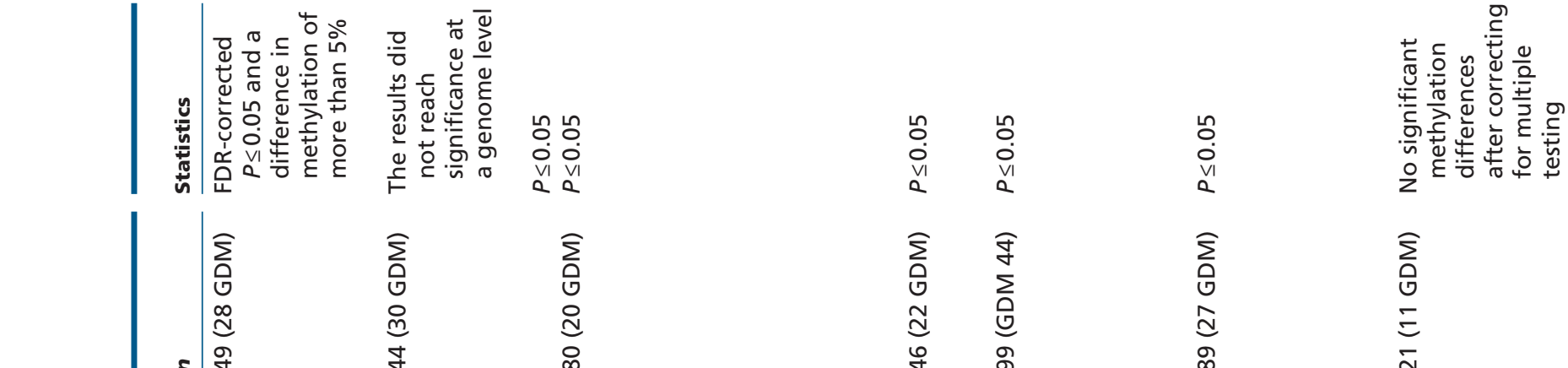

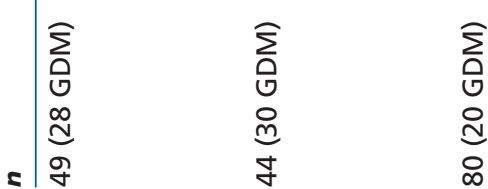

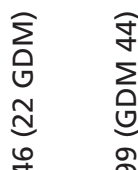

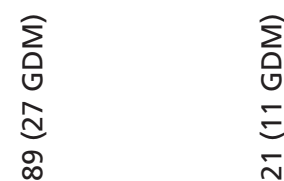

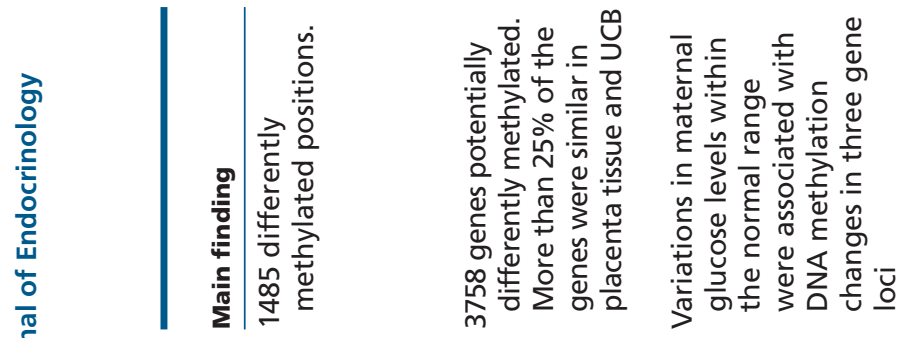

안

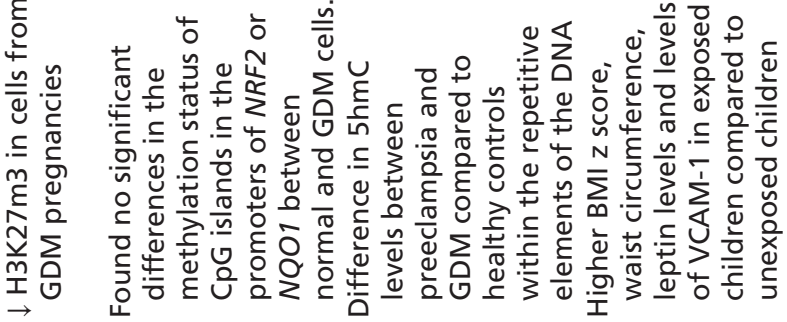

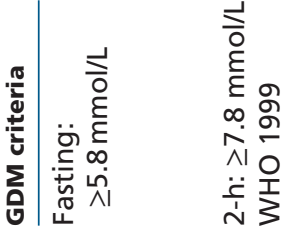

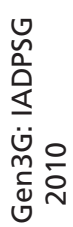

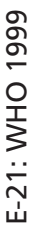

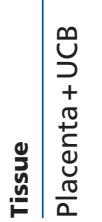

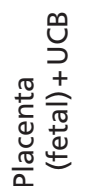

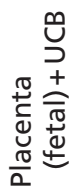

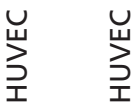

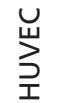

$\sum_{0}$
¿
$\bar{N}$

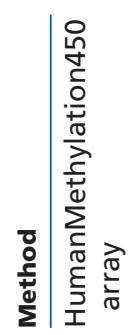

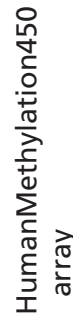

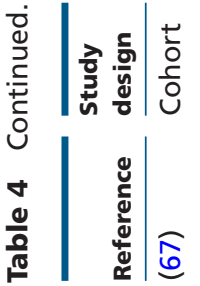

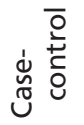

ڤิ

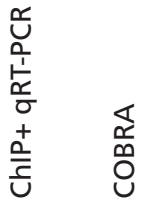

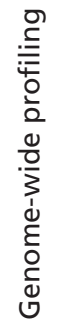

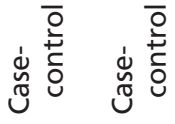

نํ.

它

हิ ู

$\widehat{\underline{B}} \quad \widehat{E}$

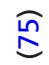

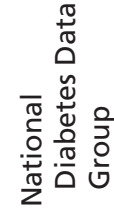

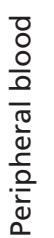




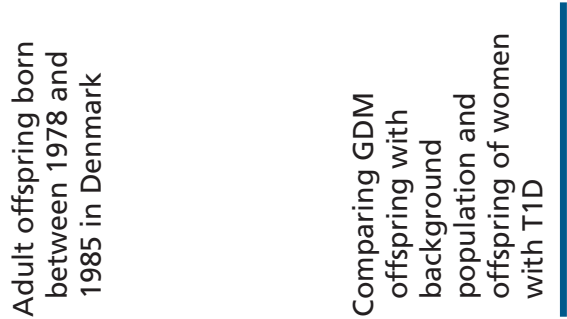

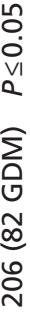

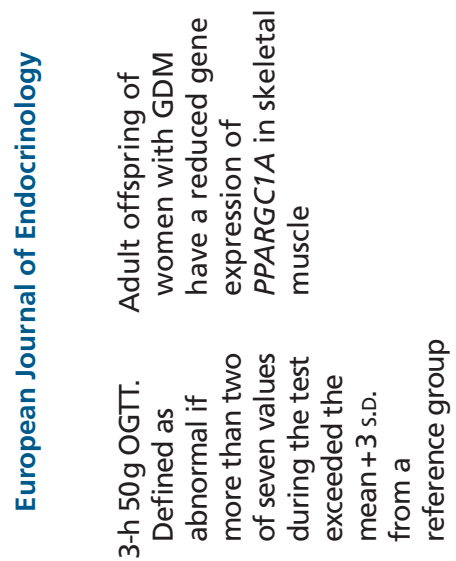

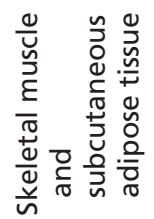

ถิ

을

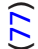

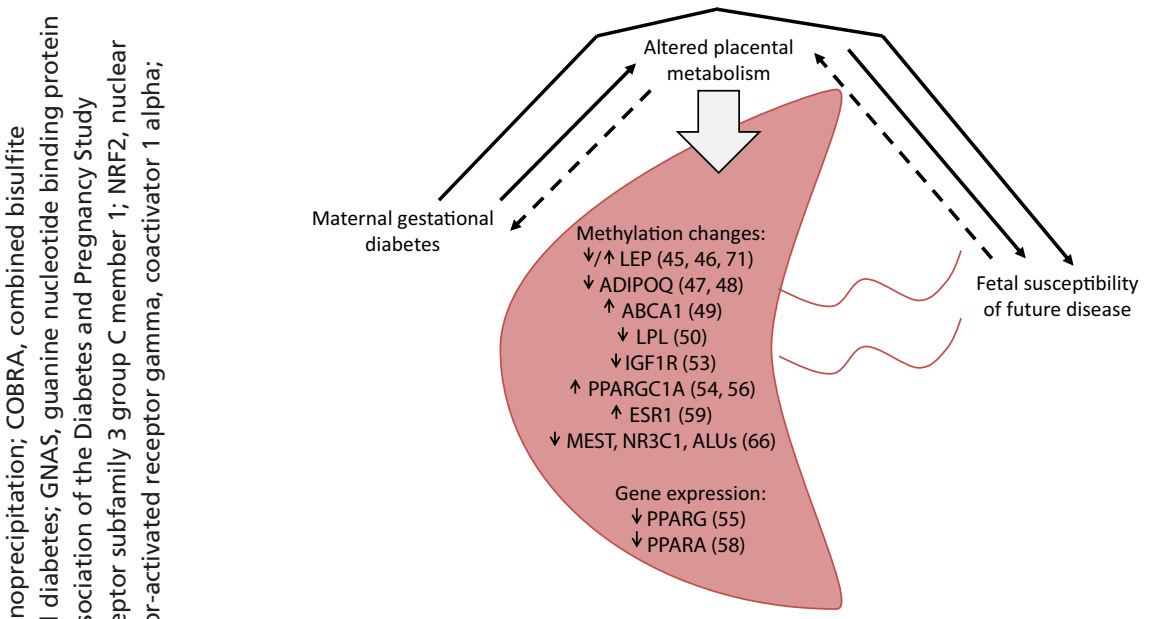

Figure 2

Conceptual framework showing how elevated maternal blood glucose may directly (black arrow) or indirectly (dashed arrow) lead to methylation changes in the placenta. As elevated maternal glucose leads to elevated delivery of glucose to the fetus, it is possible that signal molecules from the baby, such as elevated leptin levels due to increased adipose tissue, may lead to methylation changes in the placenta.

Included articles are referenced in the parenthesis.

higher degree of methylation on the maternal side. No such maternal-fetal pattern was found in the 25 nonGDM women. Furthermore, they reported a significant correlation between DNA methylation and mRNA expression in the placental tissue, but the mRNA levels did not correlate with the serum leptin levels in the third trimester. The authors argue that this may be due to the fact that leptin of placental origin constitutes only a small fraction of the total circulating maternal leptin, which was supported by their finding that the placental leptin mRNA levels account for $16 \%$ of the variance in circulating maternal leptin concentration.

\section{ADIPOQ}

A study with 98 women, 31 of which had GDM, by Bouchard and coworkers (47) showed that the mean degree of DNA methylation in the adiponectin (ADIPOQ) gene was similar on the maternal and the fetal side of the placenta. The authors also reported that a high level of maternal insulin resistance in the second and third trimester was associated with lower DNA methylation of the $A D I P O Q$ gene on the maternal side. This association was strengthened after adjustment for circulating levels of adiponectin. The authors concluded that these changes 
could mirror changes that take place in maternal adipose tissue. In a brief report in 2013 on the same material (48), it was reported that fetal methylation of $A D I P O Q$ decreased with maternal blood glucose levels.

\section{ABCA1}

Furthermore, a study in 26 GDM women and 74 healthy controls, also from the Bouchard group (49), showed a negative correlation between DNA methylation of the ATP-binding cassette transporter A1 (ABCA1) gene on the maternal side of the placenta and high-density lipoprotein cholesterol (HDL-C) levels, and a similar positive correlation with glucose levels 2-h after OGTT. On the fetal side, DNA methylation of $A B C A 1$ was negatively correlated with cord blood triglycerides. Both fetal and maternal variability in DNA methylation was associated with ABCA1 mRNA levels. Further, maternal glucose levels were negatively correlated with DNA methylation of $A B C A 1$ in cord blood.

\section{LPL}

In another study on placentas of 126 women (50), Bouchards group reported methylation of the lipoprotein lipase $(L P L)$ gene in fetal placental tissue, which showed lower methylation in 27 GDM pregnancies compared to that in 99 controls. mRNA analysis showed 1.6-fold higher expression of $L P L$ in GDM vs non-GDM women.

\section{Other genes}

In a study of 30 women with GDM compared to 14 controls (51), the Bouchard group reported that 3271 genes in placenta were nominally differentially methylated. Of these, $16 \mathrm{CpG}$ sites were validated using BSP. Replication in an independent study was unsuccessful (52). Still, they found that variations in maternal 2-h OGTT glucose levels within the normal range were associated with DNA methylation changes in the placental genes bromodomain-containing 2 (BRD2) and low-density lipoprotein receptor-related protein $1 \mathrm{~B}$ (LRP1B). Additionally, the Bouchard group reported from a study of 140 placentas (53) on the insulin-like growth factors (IGF) system, where they replicated some results from an earlier epigenome-wide association studies (EWAS study) (51). Exposure to intrauterine hyperglycemia was associated with changes in DNA methylation of insulinlike growth factor 1 receptor (IGF1R) and insulin-like growth factor-binding protein 3 (IGFBP3) in placenta of women in one of the examined cohorts; however, only the IGF1R results were replicated in the second cohort.

In a study of 305 women in two Canadian cohorts (54), the Bouchard group assessed the methylation levels in the PR domain containing 16 (PRDM16), bone morphogenetic protein 7 (BMP7), C-terminal-binding protein $2(C T B P 2)$ and peroxisome proliferator-activated receptor gamma, coactivator 1 alpha (PPARGC1A) gene loci. They found that higher degree of DNA methylation in the PPARGC1A gene locus was associated with higher second-trimester fasting glucose levels and 2-h post-OGTT glycaemia. Higher fasting glucose levels in the second and third trimester were associated with lower degree of DNA methylation in the PRDM16 gene locus. Higher 2-h postOGTT glucose levels were also associated with lower $B M P 7$ DNA methylation levels. Furthermore, BMP7 and PRDM16 DNA methylation was lower in placentas exposed to GDM compared to those not exposed. Lower degree of DNA methylation in PRDM16 and higher in PPARGC1A were associated with higher cord blood leptin levels; however, only the PRDM16 findings remained significant after adjustment for maternal glycaemia. Mediation analysis suggested that PPARGC1A mediated the link between the exposure to maternal glycaemia in the second trimester and the cord blood leptin levels.

A study on placentas from 6 women with dietary controlled GDM, six with GDM controlled by medication and matched controls (55) reported a role of miR-143 in mitochondrial function and glucose metabolism. The medication group had a significant reduction in miR143 expression compared to that in the other groups. Lower protein levels of peroxisome proliferator-activated receptor gamma (PPAR- $\gamma$ ), peroxisome proliferatoractivated receptor gamma and coactivator 1 alpha (PGC$1 \alpha)$ known to be downstream of miR-143 were observed in both GDM groups compared to controls. Moreover, the authors reported reduced mitochondrial function and increased glycolysis in the placentas from the medicated GDM cases and showed that overexpression of miR143 could partly rescue mitochondrial function and reduce glycolytic enzymes in the trophoblast cells from medication-treated placentas. However, it is difficult to assess the impact of different GDM treatments on these results.

A candidate gene study in placentas of 58 pregnancies with uncomplicated Cesarean delivery (56) showed that maternal glucose levels were positively associated with fetal-placental DNA methylation of PPARGC1A gene. Another study of 85 Chinese women with Cesarean 
delivery, from a different group (57), explored the differences in placental methylation in GDM patients and preeclampsia patients compared to that in controls. They found that 10424 loci were differently methylated in GDM women compared to those in controls. Validation in four genes was performed on 85 placentas by BSP. Here, they found hypermethylation in the gene for glucose transporter 3 (GLUT3) and hypomethylation in the peroxisome proliferator-activated receptor alpha (PPARA), retinol-binding protein 4 (RBP4) and resistin (RETN).

A study in placentas from 40 GDM women and 40 controls (58) revealed an upregulation of miR-518d in GDM. The miR-518d was shown to have a binding site on PPARA mRNA, and further that an increase in miR$518 \mathrm{~d}$ correlated with a decrease in protein level of PPAR $\alpha$. It is important to note that women with GDM had a significantly greater BMI and larger offspring than the control group. As PPARs are fatty acid receptors important in lipid homeostasis, it is unclear if the findings are due to GDM or the higher BMI.

A study of 40 women with GDM compared to 40 controls (59) indicated that expression of the placental estrogen receptor $\alpha(E R \alpha)$, a possibly important regulator of glucose metabolism in adipose and muscle tissue, was upregulated in extravillous trophoblasts (EVT). The study further demonstrated a downregulation of ERo in decidual vessels in pregnancies with a male fetus. The paper showed that mRNA for the ER $\alpha$-encoding gene (ESR1) was increased in GDM decidua compared to that for controls and that the gene promoter was only methylated in control placentas.

A study on placentas from 193 GDM pregnancies compared to 202 normal pregnancies (60) showed an upregulation of miR-98. miR-98 overexpression was positively associated with global methylation. It was further reported that miR-98 could inhibit the expression of methyl-CpG-binding protein 2 (MECP2) and further reduce the expression of transient receptor potential cation channel subfamily C member 3 (TRPC3) mRNA and protein, involved in glucose uptake.

A study of placenta tissue from 30 women (61) showed differentially expressed miRNA between cases and controls target genes involved in the epidermal growth factor receptor/phosphoinositide-3-kinase/Akt (EGFR/ PI3K/Akt) pathway, important in placental development and fetal growth. After performing a miRNA microarray expression study on five cases and five controls, 29 miRNA were significantly differentially expressed, 15 of the miRNA had targets associated with the EGFR/PI3K/ Akt pathway. The expression was validated in 10 cases and 10 controls, showing significant changes in 9 miRNA. miR-508-3p was found to be the most upregulated and to target a FYVE finger-containing phosphoinositide kinase (PIKFYVE), a negative regulator of EGFR signaling. The article further showed an increase in protein levels of EGFR, PI3K and phosphor-akt and a decrease in PIKfyve protein levels in placenta tissue. The authors suggested that this increase in expression of EGFR/PI3K/Akt pathway may be a mechanism that contributes to macrosomia in GDM babies.

One study of primary fetoplacental endothelial cells (fpEC) in 29 women (62) did not find an effect of GDM on mRNA expression of intercellular adhesion molecule 1 (ICAM1), vascular cell adhesion molecule 1 (VCAM1) and E-selectin (SELE). However, the total level of ICAM-1 was reduced by $31 \%$ in fpEC from 11 GDM mothers. A global DNA methylation analysis did not reveal any differences in methylation patterns between GDM and healthy controls in the ICAM1 gene. However, mir221 and mir222 were significantly upregulated in the GDM cells. These two microRNAs have previously been negatively associated with ICAM-1, suggesting a posttranscriptional regulation.

A study of 41 GDM women and 41 matched healthy controls (63) reported results on genome-wide methylation and expression in the maternal side of placentas. Although the authors identified four candidate regions with possible methylation differences between GDM and non-GDM women, the results did not remain significant when corrected for multiple testing and was not verified by pyrosequencing or in a second cohort of GDM women.

A study in 36 subjects with GDM and 40 controls (64) used tissue from chorionic villi to identify 6641 differentially methylated regions. Validation of methylation status and gene expression showed that the most significant and largest methylation difference, with $15 \%$ more in the control group, was in the GLUT3 gene. Validation in the resistin gene also showed higher methylation in the control group.

A study on mainly Caucasian women, 56 with GDM and 974 controls (65), used mass spectrometry to assess the difference in overall methylation status between the two groups. They reported significantly higher degree of methylation in GDM women compared to that in controls, also after multiple adjustments. However, it is difficult to evaluate the biological relevance as no other studies have applied the same methodology.

A study on placental tissue from 251 women (66) found that the maternally imprinted mesoderm-specific 
transcript (MEST) gene and the non-imprinted glucocorticoid receptor, nuclear receptor subfamily 3 group C member 1 (NR3C1) gene and interspersed ALU repeats were all hypomethylated in GDM pregnancies compared to those in controls. The study reported a similar methylation pattern for MEST in a group of obese adults compared to that in normal-weight controls.

A study on placenta tissue from 27 GDM and 21 healthy pregnancies (67) found 1708 differentially methylated positions $(>5 \%)$ that reached significance $(P<0.05)$ after FDR. Pathways that were differently methylated included endocytosis, mitogen-activated protein kinases (MAPK) signaling pathway, cell adhesion molecules (CAMs), focal adhesion, chemokine signaling and insulin signaling pathways. The results were not validated by BSP.

\section{Epigenetic alterations in the offspring}

\section{Cord blood}

A candidate gene study in cord blood of 168 offspring born to GDM mothers or controls (68) analyzed methylation differences in guanine nucleotide-binding protein alpha subunit (GNAS) and IGF2 gene loci. The methylation pattern for IGF2 did not differ between the two groups; however, three sites in the GNAS gene were hypermethylated in cord blood of offspring to GDM mothers. Methylation of IGF2 and H19 (non-protein coding) has been explored in another Chinese candidate study (69) of 275 women. IGF-2 showed higher expression in both placenta and umbilical cord, whereas H19 showed a lower expression in the cord blood in GDM compared to that in controls. No differences in methylation pattern were identified.

Methylation of the placenta-specific 8 (PLAC8) gene was negatively correlated with PLAC8 mRNA in endothelial colony-forming cells (ECFCs) in 37 children (70). The PLAC8 mRNA also correlated with maternal plasma glucose levels, and the methylation pattern differed between offspring of GDM and control mothers. Importantly, the findings were replicated in an independent cohort.

A study of 485 women (71) investigated the link between fasting hyperglycemia in mothers and epigenetic regulation of the leptin gene in infants. The authors found that fasting glucose levels in the 2 nd trimester correlated with the degree of methylation near the leptin gene in the cord blood of the offspring, which was correlated with leptin levels in cord blood.
A nested study in cord blood of 96 offspring (72) showed differences in the methylation pattern between offspring of GDM mothers compared to those in nonGDM controls. Offspring of GDM mothers shared some differently methylated loci with infants who had experienced intrauterine growth restriction compared to controls. Validations of some loci were performed by bisulfite mass array.

A study on placental tissue from 251 women (66) found that the maternally imprinted mesodermspecific transcript (MEST) gene and the non-imprinted glucocorticoid receptor (NR3C1) gene and interspersed ALU repeats were all hypomethylated in GDM pregnancies compared to those in controls. The study reported a similar methylation pattern for MEST in a group of obese adults compared to that in normal-weight controls.

A study on cord blood from 27 GDM exposed and 21 unexposed offspring (67) found 1485 differentially methylated positions $(>5 \%)$ that reached significance $(P<0.05)$ after FDR. Methylation differences were seen in pathways related to endocytosis, MAPK signaling pathway, focal adhesion, chemokine signaling and Wnt signaling pathway. The results were not validated by BSP.

In a study of 30 pregnancies with GDM compared to 14 controls (51), the Bouchard group reported that 3758 genes were potentially differently methylated; however, none was genome-wide significant. Furthermore, they reported that more than $25 \%$ of the genes that were differentially methylated were similarly modified in both placenta and cord blood. Pathway analysis showed that these genes are involved in immunological, metabolic and endocrine system disorders. Three CpG sites in cord blood samples were validated using BSP, whereas replication in an independent cohort of 80 women was unsuccessful (52). Nevertheless, they found that variations in maternal 2-h OGTT glucose levels within the normal range were associated with DNA methylation changes in the genes calcium voltage-gated channel subunit alpha 1D (CACNA1D) and LRP1B in cord blood.

\section{HUVEC}

Our search yielded three studies that used human umbilical cord vein endothelial cells (HUVEC) to study epigenetic alterations in GDM vs non-GDM pregnancies.

One study in cells from 22 GDM pregnancies compared to that in 24 healthy controls (73) looked at miRNA-101 and histone methyltransferase enhancer of zester homolog-2 (EZH2), important for H3K27me3. 
The study found an increase in miRNA-101 expression and a decrease in EZH2- $\beta$ expression and a subsequent decrease in $\mathrm{H} 3 \mathrm{~K} 27 \mathrm{~m} 3$ in cells from GDM pregnancies compared to those in controls.

One article reported no differences in methylation between cells from 44 GDM women and 55 healthy pregnancies (74) in the promoter of nuclear factorlike 2 (NRF2) or $\mathrm{NAD}(\mathrm{P}) \mathrm{H}$ dehydrogenase 1 (NQO1). However, the expression of the proteins involved in redox homeostasis was significantly altered in cells from GDM subjects.

Investigation of $5 \mathrm{hmC}$ in $27 \mathrm{GDM}$ and 62 healthy controls (75) found a significant difference within the repetitive elements of the DNA. However, the statistics are not well described in the methodology, and it is not clear what methods have been used.

\section{Children and adults}

One study compared findings in peripheral blood from offspring (age 8-12 years) of women with GDM to nonGDM controls (76). This small study of 21 subjects, found significantly higher BMI z-score, waist circumference, leptin levels and levels of VCAM1 in exposed children compared to those in unexposed children. The authors reported 84 gene regions that were differently methylated at a significance level of $P<0.005$. The results do not seem to be validated by BSP.

A study of skeletal muscle and subcutaneous adipose tissue investigated methylation changes in 260 adult offspring exposed to intrauterine hyperglycemia (77) by diet-treated GDM mothers. Offspring of GDM mothers not only had higher plasma glucose levels during an OGTT and higher diastolic blood pressure but also had 40\% lower expression of PPARGC1A in skeletal muscle compared to that in the background population. No differences in PPARGC1A DNA methylation were found between the groups; however, mean PPARGC1A DNA methylation was significantly associated with adult offspring BMI and HOMA-IR in the GDM group.

\section{Studies too small to include}

Five studies found during our initial searches had less than $10 \mathrm{GDM}$ cases and were too small for inclusion, as this small study size makes it difficult to conclude on the biological relevance of these findings. Differentially methylated sites were reported between GDM and nonGDM women in peripheral blood cells and placentas
$(78,79,80)$. Differential methylation at 127 genes (81) as well as regulation of miRNA-16 influencing mRNA expression of cyclooxygenase 2 (COX2) was reported in HUVECs (82).

\section{Discussion}

In this systematic review of epigenetic alterations in mother and offspring subjected to gestational diabetes, we found that a number of epigenetic differences have been reported in mothers with GDM and their offspring compared with healthy controls. However, the current evidence is insufficient to conclude that the reported differences play a substantial biological role with clinical implications. This is first and foremost due to the fact that most of the studies were small with limited power. Furthermore, comparing the findings in different studies is challenging due to differences in methodology, populations, study power and tissues studied.

Unfortunately, the two genome-wide DNA methylation studies using maternal WBC carried out thus far $(40,78)$ were too small for solid conclusions on methylation differences between GDM cases and controls. However, studies on miRNA in GW 16-19 $(41,42)$ suggest different expression of some miRNAs between women who later developed GDM and women who did not, suggesting their possible use as predictive markers. Early prediction of GDM may help identify risk pregnancies before regular OGTT is performed in weeks $24-28$. Some studies have tried intervention at this time point, and although no differences between the intervention group and normal care group have been observed on serious complications, such as stillbirth, a dietary intervention where women monitored their blood glucose reduced mean birth weight, neonatal fat mass as well as frequency of Cesarean delivery and shoulder dystocia $(83,84)$. Also, a Finnish RCT demonstrated that moderate lifestyle intervention can reduce the incidence of GDM with 39\% in a high-risk population of women with a BMI $\geq 30$ or a history of GDM (85). Thus, early prediction of GDM may reduce the negative outcomes, as well as prevent epigenetic alterations influencing the metabolism of the offspring.

The results from the 16 genome-wide methylation studies suggest that GDM may be associated with methylation changes in peripheral blood, placenta and umbilical cord. However, the sample sizes were small, and many of the findings did not reach significance after correction for multiple testing. Furthermore, there might 
be reporting bias, as we do not know how many studies with negative results exist and remain unpublished. Larger studies are needed to provide a complete picture of the methylation changes related to maternal hyperglycemia.

However, there are some conclusive findings. GDM seems to be associated with methylation changes in the leptin gene $(45,46)$. This adipokine, secreted by the placenta during pregnancy, plays a role in energy balance by inhibiting the secretion of insulin and stimulating glucose transport (86). Furthermore, it has previously been shown that demethylation of the leptin promoter stimulates gene transcription in adipocytes (87). Leptin is therefore an interesting candidate gene for further studies in mother and child. Adiponectin is another important adipokine known to play a role in glucose metabolism (86), and methylation changes in the gene seems to be associated with GDM (47), suggesting that exposure to GDM in utero may alter adiponectin expression in the offspring. Furthermore, changes in methylation patterns of $A B C A 1$, a transporter of cholesterol from cells to apolipoproteins A1, and a contributor to the formation of HDL (88), was observed in placenta and replicated in cord blood. As the ABCA1 gene is highly expressed in placenta and is important for maternal-fetal cholesterol transport at the maternal side $(89,90)$, a potential change in expression could lead to altered energy supply to the fetus. Another important molecule in the lipid metabolism is lipoprotein lipase, which contributes to transfer of free fatty acids from maternal lipoproteins to the fetus (91), and as mentioned previously, fetal placental tissue from mothers with GDM has a lower DNA methylation and a higher mRNA expression of $L P L$ (50).

PGC-1 $\alpha$ encoded by the PPARGC1A gene is an important coactivator of proteins involved in energy metabolism (92). Several studies have studied the methylation pattern of this gene and showed a positive association between fetal DNA methylation and maternal glucose levels $(54,56)$. In addition, adult offspring of GDM mothers had about 40\% lower gene expression of PPARGC1A mRNA in skeletal muscle compared to that in controls (77).

The GDM prevalence is associated with ethnicity, socioeconomic status as well as age, BMI and parity. A GDM prevalence of up to $42 \%$ has been reported in some ethnic populations with the new WHO 2013/IADPSG criteria (93). These factors may just as well influence the epigenetic modifications in different populations, and they need to be carefully studied. As GDM women often are more overweight than non-GDM women, one particularly important issue is the effect of the epigenetic modifications of glycemia itself and the effects of obesity per se. Moreover, the use of BMI across ethnicities represents a challenge as Asians have been found to have a higher amount of adipose tissue relative to their BMI than whites (94). They also have an increased risk of diabetes at the same BMI $(95,96)$. The studies reviewed here include a variety of different ethnicities and the underlying mechanisms for different methylation pattern could be a reflection of different body compositions. Furthermore, the different GDM diagnostic criteria used can lead to some women being classified as healthy in one study and as GDM in another study. Different factors seem to influence fasting and postprandial glucose and thereby impaired fasting glycemia (IFG) and impaired glucose tolerance (IGT) - IFG is predominantly thought to be related to age and a family history of diabetes, whereas IGT on the other hand has been associated with physical inactivity, unhealthy diet and short stature $(97,98)$. Both IGT and IFG are insulin-resistant states, but differ in site of insulin resistance. IFG is characterized mainly by hepatic insulin resistance and defective early insulin secretion, while individuals with IGT have normal to slightly reduced hepatic insulin sensitivity and higher muscle insulin resistance $(99,100)$. It is possible that the discrepancy in results presented in this review could be explained by differences in GDM criteria, representing different populations studied.

Studies of many different tissues have been included in this review. As methylation patterns can be tissue specific $(101,102,103)$, one must take into account the possible implication of methylation changes in the different tissues. Furthermore, as placenta has both a maternal and fetal side, differences are likely to occur within this organ. In addition, cellular composition is known to explain much of the observed variability in DNA methylation in a heterogeneous tissue (104) such as the placenta. To our knowledge, none of the placenta or WBC studies have been performed on single cells, with the exception of one on primary fetoplacental endothelial cells (62) and one studying extravillous trophoblasts (59). This may explain variation in results observed in, for example, the leptin studies. Although several of the studies in this review report epigenetic changes in peripheral blood, these changes do not necessarily lead to the same functional effects in blood as in placenta, adipose or pancreas tissue. Studies of pancreatic tissue have shown changes in epigenetic signaling between diabetic and non-diabetic in humans and rats $(105,106)$, as well as in adipose tissue (107). Interestingly, one study reporting different methylations between diabetic and non-diabetic 
patients found methylation differences in pancreatic islet cells, but could not find these differences in blood cells (108). Moreover, a systematic review on blood cells, muscle, adipose and placenta tissue did not find any general overlap of epigenetic modifications between the tissues (109). More studies on the same tissue and same cell types are needed to compare results and assess their reproducibility. Furthermore, additional studies on tissue biopsies in offspring would be beneficial to investigate potential functional changes, as well as to assess how methylation changes on maternal and fetal side of the placenta may influence offspring of GDM mothers.

Furthermore, it is important to consider the methods used in the studies when interpreting the result, as the quality of the technologies used varies greatly. A review by Kurdyukov and Bullock (110) gives an overview of the methods used divided into digestion-based assays followed by PCR, bisulfite conversion, whole genome methylation profiling and search for differentially methylated regions. When candidate genes are not known, wholegenome methylation profiling or search for differentially methylated regions is an appropriate strategy. The most common methods used in this review are array or bead hybridization for determining differentially methylated regions in unknown candidate genes and bisulfite pyrosequencing for known candidate genes. Methods for the individual studies can be viewed in Tables 2, 3 and 4. As the functional effect of DNA methylation occurs within gene promoter regions, enhancer regulatory elements and 3'-UTRs assays focusing on these regions can save both time any money, but it is important to be aware that it is reported that up to $6 \%$ (111) of the probes could give false-positive results due to cross-reactivity. The result from methods such as HumanMethylation450 Bead Chip array from Illumina should always be validated. Bisulfite sequencing is considered the gold standard for differentially methylated regions. When studying methylation in already known candidate genes or when validating potentially differentially methylated regions, bisulfite conversion followed by methylation-specific PCR or pyrosequencing would be appropriate methods.

Despite a number of methodological problems, most of the cited studies conclude that GDM is associated with epigenetic alterations. However, we cannot exclude the possibility that this could be due to bias or random chance, as many of the studies do not correct for multiple testing. It is also important to keep in mind that we do not know if the potential epigenetic changes are causal for GDM or alterations in the offspring, if the epigenetic changes are caused by underlying genetic factors, and is only a mediator, or possibly that a combination of genetics and environmental components causes GDM and influences the offspring.

Many of the genes that are reported to be differently regulated are involved in energy and/or glucose metabolism and could influence the weight regulation and glucose metabolism of the offspring. GDM may contribute to the accumulation of obesity in future generations by increasing the risk of adiposity and/or diabetes in the offspring. More studies are needed using the same GDM criteria within the different ethnic populations. By gathering more information about epigenetic alterations, we may be enabled to earlier detection of risk individuals and hence more appropriate preventive actions.

\section{Conclusion/further research}

In this systematic review, we have reviewed the current evidence and shown sizable knowledge gaps in the field of epigenetics and gestational diabetes. Methodological problems, diagnostic criteria and differences in ethnic composition, together with a limited number of studies and small sample sizes preclude final conclusions at this stage. The need for global DNA methylation studies with larger statistical power is apparent. Although the reviewed studies suggest that epigenetic alterations can be found both in women with GDM and their offspring, some of the findings may be due to chance or bias due to low study power. Studies with higher study power in multi-ethnic populations are required to address these questions. Moreover, most studies are based on chips that have limited coverage or restricted to a few sites, whereas it has been shown that methylation acts through a signature of multiple $\mathrm{CpG}$ sites in tandem, rather than independent CpGs. Whole-genome sequencing would go a long way in identifying such signatures. Further research is warranted to increase the knowledge in this field and possibly determine new routes for tailored interventions in risk populations.

\section{Declaration of interest}

The authors declare that there is no conflict of interest that could be perceived as prejudicing the impartiality of this review.

\section{Funding}

This review did not receive any specific grant from any funding agency in the public, commercial or not-for-profit sector. G H M has a PhD grant from the South-Eastern Norway Regional Health Authority (Grant number 2015008). 


\section{Author contribution statement}

G H M performed the search and scanned through all abstracts, read all papers and wrote the manuscript. C S scanned through all abstracts and critically revised the manuscript. $R$ P critically revised the manuscript. L S critically revised the manuscript. L G critically revised the manuscript. E Q read half of the papers and critically revised the manuscript. $K$ I B read half of the papers and critically revised the manuscript.

\section{References}

1 Catalano PM. The impact of gestational diabetes and maternal obesity on the mother and her offspring. Journal of Developmental Origins of Health and Disease 20101 208-215. (doi:10.1017/ S2040174410000115)

2 Gillman MW, Rifas-Shiman S, Berkey CS, Field AE \& Colditz GA. Maternal gestational diabetes, birth weight, and adolescent obesity. Pediatrics 2003111 e221-e226. (doi:10.1542/ peds.111.3.e221)

3 Kim C, Newton KM \& Knopp RH. Gestational diabetes and the incidence of type 2 diabetes. A Systematic Review $2002 \mathbf{2 5}$ 1862-1868. (doi:10.2337/diacare.25.10.1862)

4 Damm P, Houshmand-Oeregaard A, Kelstrup L, Lauenborg J, Mathiesen ER \& Clausen TD. Gestational diabetes mellitus and long-term consequences for mother and offspring: a view from Denmark. Diabetologia 201659 1396-1399. (doi:10.1007/s00125016-3985-5)

5 Watanabe RM. Inherited destiny? Genetics and gestational diabetes mellitus. Genome Medicine 20113 18. (doi:10.1186/ gm232)

6 Lowe WL, Scholtens DM, Sandler V \& Hayes MG. Genetics of gestational diabetes mellitus and maternal metabolism. Current Diabetes Reports 201616 15. (doi:10.1007/s11892-015-0709-z)

7 Forsdahl A. Are poor living conditions in childhood and adolescence an important risk factor for arteriosclerotic heart disease? British Journal of Preventive and Social Medicine 197731 91-95.

8 Hales CN \& Barker DJ. Type 2 (non-insulin-dependent) diabetes mellitus: the thrifty phenotype hypothesis. Diabetologia 199235 595-601. (doi:10.1007/BF00400248)

9 Roseboom TJ, van der Meulen JH, Ravelli AC, Osmond C, Barker DJ \& Bleker OP. Effects of prenatal exposure to the Dutch famine on adult disease in later life: an overview. Molecular and Cellular Endocrinology 2001185 93-98. (doi:10.1016/S03037207(01)00721-3)

10 Gillman MW. Developmental origins of health and disease. New England Journal of Medicine 2005353 1848-1850.

11 Godfrey KM \& Barker DJ. Fetal nutrition and adult disease. American Journal of Clinical Nutrition 200071 1344s-1352s.

12 Barker DJ, Gluckman PD, Godfrey KM, Harding JE, Owens JA \& Robinson JS. Fetal nutrition and cardiovascular disease in adult life. Lancet $1993 \mathbf{3 4 1}$ 938-941. (doi:10.1016/01406736(93)91224-A)

13 Seghieri G, Anichini R, De Bellis A, Alviggi L, Franconi F \& Breschi MC. Relationship between gestational diabetes mellitus and low maternal birth weight. Diabetes Care 200225 1761-1765. (doi:10.2337/diacare.25.10.1761)

14 McCance DR, Pettitt DJ, Hanson RL, Jacobsson LT, Knowler WC $\&$ Bennett PH. Birth weight and non-insulin dependent diabetes: thrifty genotype, thrifty phenotype, or surviving small baby genotype? BMJ 1994308 942-945. (doi:10.1136/ bmj.308.6934.942)

15 Horikoshi M, Yaghootkar H, Mook-Kanamori DO, Sovio U, Taal HR, Hennig BJ, Bradfield JP, St Pourcain B, Evans DM, Charoen $\mathrm{P}$ et al. New loci associated with birth weight identify genetic links between intrauterine growth and adult height and metabolism. Nature Genetics 201345 76-82. (doi:10.1038/ ng.2477)

16 Hattersley AT \& Tooke JE. The fetal insulin hypothesis: an alternative explanation of the association of low birthweight with diabetes and vascular disease. Lancet 1999353 1789-1792.

17 Schlemm L, Haumann HM, Ziegner M, Stirnberg B, Sohn A, Alter M, Pfab T, Kalache KD, Guthmann F \& Hocher B. New evidence for the fetal insulin hypothesis: fetal angiotensinogen M235T polymorphism is associated with birth weight and elevated fetal total glycated hemoglobin at birth. Journal of Hypertension 201028 732-739. (doi:10.1097/ HJH.0b013e328336a090)

18 Hattersley AT, Beards F, Ballantyne E, Appleton M, Harvey R \& Ellard S. Mutations in the glucokinase gene of the fetus result in reduced birth weight. Nature Genetics 199819 268-270. (doi:10.1038/953)

19 Lawlor DA. The Society for Social Medicine John Pemberton Lecture 2011. Developmental overnutrition - an old hypothesis with new importance? International Journal of Epidemiology 2013 42 7-29. (doi:10.1093/ije/dys209)

20 Prasad RB \& Groop L. Genetics of type 2 diabetes-pitfalls and possibilities. Genes 20156 87-123. (doi:10.3390/genes6010087)

21 Zhang C, Bao W, Rong Y, Yang H, Bowers K, Yeung E \& Kiely M. Genetic variants and the risk of gestational diabetes mellitus: a systematic review. Human Reproduction Update 201319 376-390. (doi:10.1093/humupd/dmt013)

22 Zhang Y, Sun CM, Hu XQ \& Zhao Y. Relationship between melatonin receptor $1 \mathrm{~B}$ and insulin receptor substrate 1 polymorphisms with gestational diabetes mellitus: a systematic review and meta-analysis. Scientific Reports 201446113. (doi:10.1038/srep06113)

23 Wu L, Cui L, Tam WH, Ma RC \& Wang CC. Genetic variants associated with gestational diabetes mellitus: a meta-analysis and subgroup analysis. Scientific Reports 20166 30539. (doi:10.1038/ srep30539)

24 Lin PC, Lin WT, Yeh YH \& Wung SF. Transcription factor 7-like 2 (TCF7L2) rs7903146 polymorphism as a risk factor for gestational diabetes mellitus: a meta-analysis. PLoS ONE 201611 e0153044. (doi:10.1371/journal.pone.0153044)

25 Chang S, Wang Z, Wu L, Lu X, Shangguan S, Xin Y, Li L \& Wang L. Association between TCF7L2 polymorphisms and gestational diabetes mellitus: a meta-analysis. Journal of Diabetes Investigation 2017 In press. (doi:10.1111/jdi.12612)

26 Cui J, Xu X, Yin S, Chen F, Li P \& Song C. Meta-analysis of the association between four CAPN10 gene variants and gestational diabetes mellitus. Archives of Gynecology and Obstetrics 2016294 447-453. (doi:10.1007/s00404-016-4140-8)

27 Nolan CJ, Damm P \& Prentki M. Type 2 diabetes across generations: from pathophysiology to prevention and management. Lancet 2011378 169-181. (doi:10.1016/S01406736(11)60614-4)

28 El Hajj N, Schneider E, Lehnen H \& Haaf T. Epigenetics and life-long consequences of an adverse nutritional and diabetic intrauterine environment. Reproduction 2014148 R111-R120. (doi:10.1530/REP-14-0334)

29 Dabelea D, Hanson RL, Lindsay RS, Pettitt DJ, Imperatore G, Gabir MM, Roumain J, Bennett PH \& Knowler WC. Intrauterine exposure to diabetes conveys risks for type 2 diabetes and obesity: a study of discordant sibships. Diabetes 200049 2208-2211. (doi:10.2337/diabetes.49.12.2208)

30 McLean M, Chipps D \& Cheung NW. Mother to child transmission of diabetes mellitus: does gestational diabetes program Type 2 diabetes in the next generation? Diabetic Medicine 200623 1213-1215. (doi:10.1111/j.1464-5491.2006.01979.x) 
31 Harder T, Franke K, Kohlhoff R \& Plagemann A. Maternal and paternal family history of diabetes in women with gestational diabetes or insulin-dependent diabetes mellitus type I. Gynecologic and Obstetric Investigation 200151 160-164. (doi:10.1159/000052916)

32 Hocher B. More than genes: the advanced fetal programming hypothesis. Journal of Reproductive Immunology 2014 104-105 8-11. (doi:10.1016/j.jri.2014.03.001)

33 Reichetzeder C, Dwi Putra SE, Li J \& Hocher B. Developmental origins of disease - crisis precipitates change. Cellular Physiology and Biochemistry 201639 919-938. (doi:10.1159/000447801)

34 Hocher B, Haumann H, Rahnenfuhrer J, Reichetzeder C, Kalk P, Pfab T, Tsuprykov O, Winter S, Hofmann U, Li J et al. Maternal eNOS deficiency determines a fatty liver phenotype of the offspring in a sex dependent manner. Epigenetics $2016 \mathbf{1 1}$ 539-552. (doi:10.1080/15592294.2016.1184800)

35 Hernando-Herraez I, Garcia-Perez R, Sharp AJ \& Marques-Bonet T. DNA methylation: insights into human evolution. PLoS Genetics 201511 e1005661. (doi:10.1371/journal.pgen.1005661)

36 Guo JU, Su Y, Zhong C, Ming G \& Song H. Hydroxylation of 5-methylcytosine by TET1 promotes active DNA demethylation in the adult brain. Cell 2011145 423-434. (doi:10.1016/j. cell.2011.03.022)

37 Martin C \& Zhang Y. The diverse functions of histone lysine methylation. Nature Reviews Molecular Cell Biology 20056 838-849. (doi:10.1038/nrm1761)

38 Zhao S \& Liu M-F. Mechanisms of microRNA-mediated gene regulation. Science in China Series C: Life Sciences 200952 1111-1116. (doi:10.1007/s11427-009-0152-y)

39 Liberati A, Altman DG, Tetzlaff J, Mulrow C, Gotzsche PC, Ioannidis JP, Clarke M, Devereaux PJ, Kleijnen J \& Moher D. The PRISMA statement for reporting systematic reviews and meta-analyses of studies that evaluate health care interventions: explanation and elaboration. PLoS Medicine 20096 e1000100. (doi: 10.1371/journal.pmed.1000100)

40 Wu P, Farrell WE, Haworth KE, Emes RD, Kitchen MO, Glossop JR, Hanna FW \& Fryer AA. Maternal genome-wide DNA methylation profiling in gestational diabetes shows distinctive diseaseassociated changes relative to matched healthy pregnancies. Epigenetics 2016 Epub ahead of print . (doi:10.1080/15592294. 2016.1166321)

41 Zhu Y, Tian F, Li H, Zhou Y, Lu J \& Ge Q. Profiling maternal plasma microRNA expression in early pregnancy to predict gestational diabetes mellitus. International Journal of Gynecology and Obstetrics 2015130 49-53. (doi:10.1016/j. ijgo.2015.01.010)

42 Zhao C, Dong J, Jiang T, Shi Z, Yu B, Zhu Y, Chen D, Xu J, Huo R, Dai J et al. Early second-trimester serum miRNA profiling predicts gestational diabetes mellitus. PLoS ONE 20116 e23925. (doi:10.1371/journal.pone.0023925)

43 Michalczyk AA, Dunbar JA, Janus ED, Best JD, Ebeling PR, Ackland MJ, Asproloupos D \& Ackland ML. Epigenetic markers to predict conversion from gestational diabetes to type 2 diabetes. Journal of Clinical Endocrinology and Metabolism 2016101 2396-2404. (doi:10.1210/jc.2015-4206)

44 Shi Z, Zhao C, Guo X, Ding H, Cui Y, Shen R \& Liu J. Differential expression of microRNAs in omental adipose tissue from gestational diabetes mellitus subjects reveals miR-222 as a regulator of ERalpha expression in estrogen-induced insulin resistance. Endocrinology 2014155 1982-1990. (doi:10.1210/ en.2013-2046)

45 Lesseur C, Armstrong DA, Paquette AG, Li Z, Padbury JF $\&$ Marsit CJ. Maternal obesity and gestational diabetes are associated with placental leptin DNA methylation. American Journal of Obstetrics and Gynecology 2014211 654.e651-654.e659. (doi:10.1016/j.ajog.2014.06.037)
46 Bouchard L, Thibault S, Guay SP, Santure M, Monpetit A, St-Pierre J, Perron P \& Brisson D. Leptin gene epigenetic adaptation to impaired glucose metabolism during pregnancy. Diabetes Care 201033 2436-2441. (doi:10.2337/dc10-1024)

47 Bouchard L, Hivert MF, Guay SP, St-Pierre J, Perron P \& Brisson D. Placental adiponectin gene DNA methylation levels are associated with mothers' blood glucose concentration. Diabetes 201261 1272-1280. (doi:10.2337/db11-1160)

48 Houde AA, Hivert MF \& Bouchard L. Fetal epigenetic programming of adipokines. Adipocyte 20132 41-46. (doi:10.4161/adip.22055)

49 Houde AA, Guay SP, Desgagne V, Hivert MF, Baillargeon JP, St-Pierre J, Perron P, Gaudet D, Brisson D \& Bouchard L. Adaptations of placental and cord blood ABCA1 DNA methylation profile to maternal metabolic status. Epigenetics 2013 8 1289-1302. (doi:10.4161/epi.26554)

50 Houde AA, St-Pierre J, Hivert MF, Baillargeon JP, Perron P, Gaudet D, Brisson D \& Bouchard L. Placental lipoprotein lipase DNA methylation levels are associated with gestational diabetes mellitus and maternal and cord blood lipid profiles. Journal of Developmental Origins of Health and Disease 20145 132-141. (doi:10.1017/S2040174414000038)

51 Ruchat SM, Houde AA, Voisin G, St-Pierre J, Perron P, Baillargeon JP, Gaudet D, Hivert MF, Brisson D \& Bouchard L. Gestational diabetes mellitus epigenetically affects genes predominantly involved in metabolic diseases. Epigenetics 20138 935-943. (doi:10.4161/epi.25578)

52 Houde AA, Ruchat SM, Allard C, Baillargeon JP, St-Pierre J, Perron P, Gaudet D, Brisson D, Hivert MF \& Bouchard L. LRP1B, BRD2 and CACNA1D: new candidate genes in fetal metabolic programming of newborns exposed to maternal hyperglycemia. Epigenomics 20157 1111-1122. (doi:10.2217/epi.15.72)

53 Desgagne V, Hivert MF, St-Pierre J, Guay SP, Baillargeon JP, Perron P, Gaudet D, Brisson D \& Bouchard L. Epigenetic dysregulation of the IGF system in placenta of newborns exposed to maternal impaired glucose tolerance. Epigenomics 20146 193-207. (doi:10.2217/epi.14.3)

54 Cote S, Gagne-Ouellet V, Guay SP, Allard C, Houde AA, Perron P, Baillargeon JP, Gaudet D, Guerin R, Brisson D et al. PPARGC1alpha gene DNA methylation variations in human placenta mediate the link between maternal hyperglycemia and leptin levels in newborns. Clinical Epigenetics 2016872. (doi:10.1186/s13148-016-0239-9)

55 Muralimanoharan S, Maloyan A \& Myatt L. Mitochondrial function and glucose metabolism in the placenta with gestational diabetes mellitus: role of miR-143. Clinical Science 2016130 931-941. (doi:10.1042/CS20160076)

56 Xie X, Gao H, Zeng W, Chen S, Feng L, Deng D, Qiao FY, Liao L, McCormick K, Ning Q et al. Placental DNA methylation of peroxisome-proliferator-activated receptor-gamma co-activator1alpha promoter is associated with maternal gestational glucose level. Clinical Science 2015129 385-394. (doi:10.1042/ CS20140688)

57 Liu L, Zhang X, Rong C, Rui C, Ji H, Qian YJ, Jia R \& Sun L. Distinct DNA methylomes of human placentas between preeclampsia and gestational diabetes mellitus. Cellular Physiology and Biochemistry 201434 1877-1889. (doi:10.1159/000366386)

58 Zhao C, Zhang T, Shi Z, Ding H \& Ling X. MicroRNA-518d regulates PPARalpha protein expression in the placentas of females with gestational diabetes mellitus. Molecular Medicine Reports 20149 2085-2090. (doi:10.3892/mmr.2014.2058)

59 Knabl J, Hiden U, Huttenbrenner R, Riedel C, Hutter S, Kirn V, Gunthner-Biller M, Desoye G, Kainer F \& Jeschke U. GDM alters expression of placental estrogen receptor alpha in a cell type and gender-specific manner. Reproductive Sciences 201522 1488-1495. (doi:10.1177/1933719115585147) 
60 Cao J-L, Zhang L, Li J, Tian S, Lv X-D, Wang X-Q, Su X, Li Y, Hu Y, Ma X et al. Up-regulation of miR-98 and unraveling regulatory mechanisms in gestational diabetes mellitus. Scientific Reports 20166 32268. (doi:10.1038/srep32268)

61 Li J, Song L, Zhou L, Wu J, Sheng C, Chen H, Liu Y, Gao S \& Huang W. A microRNA signature in gestational diabetes mellitus associated with risk of macrosomia. Cellular Physiology and Biochemistry 201537 243-252. (doi:10.1159/000430349)

62 Diaz-Perez FI, Hiden U, Gauster M, Lang I, Konya V, Heinemann A, Logl J, Saffery R, Desoye G \& Cvitic S. Posttranscriptional down regulation of ICAM-1 in feto-placental endothelium in GDM. Cell Adhesion and Migration 201610 18-27. (doi:10.1080/19336918.2015.1127467)

63 Binder AM, LaRocca J, Lesseur C, Marsit CJ \& Michels KB. Epigenome-wide and transcriptome-wide analyses reveal gestational diabetes is associated with alterations in the human leukocyte antigen complex. Clinical Epigenetics 2015779. (doi:10.1186/s13148-015-0116-y)

64 Rong C, Cui X, Chen J, Qian Y, Jia R \& Hu Y. DNA methylation profiles in placenta and its association with gestational diabetes mellitus. Experimental and Clinical Endocrinology and Diabetes 2015 123 282-288. (doi:10.1055/s-0034-1398666)

65 Reichetzeder C, Dwi Putra SE, Pfab T, Slowinski T, Neuber C, Kleuser B \& Hocher B. Increased global placental DNA methylation levels are associated with gestational diabetes. Clinical Epigenetics 20168 82. (doi:10.1186/s13148-016-0247-9)

66 El Hajj N, Pliushch G, Schneider E, Dittrich M, Muller T, Korenkov M, Aretz M, Zechner U, Lehnen H \& Haaf T. Metabolic programming of MEST DNA methylation by intrauterine exposure to gestational diabetes mellitus. Diabetes 201362 1320-1328. (doi:10.2337/db12-0289)

67 Finer S, Mathews C, Lowe R, Smart M, Hillman S, Foo L, Sinha A, Williams D, Rakyan VK \& Hitman GA. Maternal gestational diabetes is associated with genome-wide DNA methylation variation in placenta and cord blood of exposed offspring. Human Molecular Genetics 201524 3021-3029. (doi:10.1093/hmg/ ddv013)

68 Chen D, Zhang A, Fang M, Fang R, Ge J, Jiang Y, Zhang H, Han C, Ye $\mathrm{X}$, Huang $\mathrm{H}$ et al. Increased methylation at differentially methylated region of GNAS in infants born to gestational diabetes. BMC Medical Genetics 201415 108. (doi:10.1186/s12881014-0108-3)

69 Su R, Wang C, Feng H, Lin L, Liu X, Wei Y \& Yang H. Alteration in expression and methylation of IGF2/H19 in placenta and umbilical cord blood are associated with macrosomia exposed to intrauterine hyperglycemia. PLoS ONE 201611 e0148399. (doi:10.1371/journal.pone.0148399)

70 Blue EK, Sheehan BM, Nuss ZV, Boyle FA, Hocutt CM, Gohn CR, Varberg KM, McClintick JN \& Haneline LS. Epigenetic regulation of placenta-specific 8 contributes to altered function of endothelial colony-forming cells exposed to intrauterine gestational diabetes mellitus. Diabetes 201564 2664-2675. (doi:10.2337/db14-1709)

71 Allard C, Desgagne V, Patenaude J, Lacroix M, Guillemette L, Battista MC, Doyon M, Menard J, Ardilouze JL, Perron P et al. Mendelian randomization supports causality between maternal hyperglycemia and epigenetic regulation of leptin gene in newborns. Epigenetics 201510 342-351. (doi:10.1080/15592294.2 015.1029700)

72 Quilter CR, Cooper WN, Cliffe KM, Skinner BM, Prentice PM, Nelson L, Bauer J, Ong KK, Constancia M, Lowe WL et al. Impact on offspring methylation patterns of maternal gestational diabetes mellitus and intrauterine growth restraint suggest common genes and pathways linked to subsequent type 2 diabetes risk. FASEB Journal 201428 4868-4879. (doi:10.1096/ fj.14-255240)
73 Floris I, Descamps B, Vardeu A, Mitic T, Posadino AM, Shantikumar S, Sala-Newby G, Capobianco G, Mangialardi G, Howard L et al. Gestational diabetes mellitus impairs fetal endothelial cell functions through a mechanism involving microRNA-101 and histone methyltransferase enhancer of zester homolog-2. Arteriosclerosis, Thrombosis, and Vascular Biology 2015 35 664-674. (doi:10.1161/ATVBAHA.114.304730)

74 Cheng X, Chapple SJ, Patel B, Puszyk W, Sugden D, Yin X, Mayr M, Siow RC \& Mann GE. Gestational diabetes mellitus impairs Nrf2-mediated adaptive antioxidant defenses and redox signaling in fetal endothelial cells in utero. Diabetes 201362 4088-4097. (doi:10.2337/db13-0169)

75 Sun M, Song MM, Wei B, Gao Q, Li L, Yao B, Chen L, Lin L, Dai $\mathrm{Q}$, Zhou X et al. 5-Hydroxymethylcytosine-mediated alteration of transposon activity associated with the exposure to adverse in utero environments in human. Human Molecular Genetics 201625 2208-2219. (doi:10.1093/hmg/ddw089)

76 West NA, Kechris K \& Dabelea D. Exposure to maternal diabetes in utero and DNA methylation patterns in the offspring. Immunometabolism 20131 1-9. (doi:10.2478/immun-2013-0001)

77 Kelstrup L, Hjort L, Houshmand-Oeregaard A, Clausen TD, Hansen NS, Broholm C, Borch-Johnsen L, Mathiesen ER, Vaag AA \& Damm P. Gene expression and DNA methylation of PPARGC1A in muscle and adipose tissue from adult offspring of women with diabetes in pregnancy. Diabetes 2016. (doi:10.2337/db16-0227)

78 Enquobahrie DA, Moore A, Muhie S, Tadesse MG, Lin S \& Williams MA. Early pregnancy maternal blood DNA methylation in repeat pregnancies and change in gestational diabetes mellitus status-a pilot study. Reproductive Sciences 201522 904-910. (doi:10.1177/1933719115570903)

79 Petropoulos S, Guillemin C, Ergaz Z, Dimov S, Suderman M, Weinstein-Fudim L, Ornoy A \& Szyf M. Gestational diabetes alters offspring DNA methylation profiles in human and rat: identification of key pathways involved in endocrine system disorders, insulin signaling, diabetes signaling, and ILK signaling. Endocrinology 2015156 2222-2238. (doi:10.1210/ en.2014-1643)

80 Nomura Y, Lambertini L, Rialdi A, Lee M, Mystal EY, Grabie M, Manaster I, Huynh N, Finik J, Davey M et al. Global methylation in the placenta and umbilical cord blood from pregnancies with maternal gestational diabetes, preeclampsia, and obesity. Reproductive Sciences 201421 131-137. (doi:10.1177/1933719113492206)

81 Ambra R, Manca S, Palumbo MC, Leoni G, Natarelli L, De Marco A, Consoli A, Pandolfi A \& Virgili F. Transcriptome analysis of human primary endothelial cells (HUVEC) from umbilical cords of gestational diabetic mothers reveals candidate sites for an epigenetic modulation of specific gene expression. Genomics 2014 103 337-348. (doi:10.1016/j.ygeno.2014.03.003)

82 Di Francesco L, Dovizio M, Trenti A, Marcantoni E, Moore A, O'Gaora P, McCarthy C, Tacconelli S, Bruno A, Alberti S et al. Dysregulated post-transcriptional control of COX-2 gene expression in gestational diabetic endothelial cells. British Journal of Pharmacology 2015172 4575-4587. (doi:10.1111/bph.13241)

83 Landon MB, Spong CY, Thom E, Carpenter MW, Ramin SM, Casey B, Wapner RJ, Varner MW, Rouse DJ, Thorp JM et al. A multicenter, randomized trial of treatment for mild gestational diabetes. New England Journal of Medicine 2009361 1339-1348. (doi:10.1056/NEJMoa0902430)

84 Crowther CA, Hiller JE, Moss JR, McPhee AJ, Jeffries WS \& Robinson JS. Effect of treatment of gestational diabetes mellitus on pregnancy outcomes. New England Journal of Medicine 2005 352 2477-2486. (doi:10.1056/NEJMoa042973)

85 Koivusalo SB, Rono K, Klemetti MM, Roine RP, Lindstrom J, Erkkola M, Kaaja RJ, Poyhonen-Alho M, Tiitinen A, Huvinen E et al. Gestational diabetes mellitus can be prevented by 
lifestyle intervention: the Finnish gestational diabetes prevention study (RADIEL): a randomized controlled trial. Diabetes Care 2016 39 24-30. (doi:10.2337/dc15-0511)

86 Zavalza-Gomez AB, Anaya-Prado R, Rincon-Sanchez AR \& Mora-Martinez JM. Adipokines and insulin resistance during pregnancy. Diabetes Research and Clinical Practice $2008 \mathbf{8 0}$ 8-15 (doi:10.1016/i.diabres.2007.12.012)

87 Melzner I, Scott V, Dorsch K, Fischer P, Wabitsch M, Bruderlein S, Hasel C \& Moller P. Leptin gene expression in human preadipocytes is switched on by maturation-induced demethylation of distinct CpGs in its proximal promoter. Journal of Biological Chemistry 2002277 45420-45427. (doi:10.1074/jbc. M208511200)

88 Joyce C, Freeman L, Brewer HB Jr \& Santamarina-Fojo S. Study of ABCA1 function in transgenic mice. Arteriosclerosis, Thrombosis, and Vascular Biology 200323 965-971. (doi:10.1161/01. ATV.0000055194.85073.FF)

89 Albrecht C, Soumian S, Tetlow N, Patel P, Sullivan MH, Lakasing L, Nicolaides K \& Williamson C. Placental ABCA1 expression is reduced in primary antiphospholipid syndrome compared to pre-eclampsia and controls. Placenta $2007 \mathbf{2 8}$ 701-708. (doi:10.1016/j.placenta.2006.10.001)

90 Nikitina L, Wenger F, Baumann M, Surbek D, Korner M \& Albrecht C. Expression and localization pattern of ABCA1 in diverse human placental primary cells and tissues. Placenta 2011 32 420-430. (doi:10.1016/j.placenta.2011.03.003)

91 Larque E, Demmelmair H, Gil-Sanchez A, Prieto-Sanchez MT, Blanco JE, Pagan A, Faber FL, Zamora S, Parrilla JJ \& Koletzko B. Placental transfer of fatty acids and fetal implications. American Journal of Clinical Nutrition 201194 1908s-1913s. (doi:10.3945/ ajcn.110.001230)

92 Liang H \& Ward WF. PGC-1alpha: a key regulator of energy metabolism. Advances in Physiology Education 200630 145-151. (doi:10.1152/advan.00052.2006)

93 Jenum AK, Sommer C, Sletner L, Mørkrid K, Bærug A \& Mosdøl A. Adiposity and hyperglycaemia in pregnancy and related health outcomes in European ethnic minorities of Asian and African origin: a review. Food and Nutrition Research 201357. (doi:10.3402/fnr.v57i0.18889)

94 Wang J, Thornton JC, Russell M, Burastero S, Heymsfield S \& Pierson RN Jr. Asians have lower body mass index (BMI) but higher percent body fat than do whites: comparisons of anthropometric measurements. American Journal of Clinical Nutrition 199460 23-28.

95 Boffetta P, McLerran D, Chen Y, Inoue M, Sinha R, He J, Gupta PC, Tsugane S, Irie F, Tamakoshi A et al. Body mass index and diabetes in Asia: a cross-sectional pooled analysis of 900,000 individuals in the Asia cohort consortium. PLOS ONE 20116 e19930. (doi:10.1371/journal.pone.0019930)

96 Ntuk UE, Gill JM, Mackay DF, Sattar N \& Pell JP. Ethnic-specific obesity cutoffs for diabetes risk: cross-sectional study of 490,288 UK biobank participants. Diabetes Care 201437 2500-2507. (doi:10.2337/dc13-2966)

97 Faerch K, Vaag A, Witte DR, Jorgensen T, Pedersen O \& BorchJohnsen K. Predictors of future fasting and 2-h post-OGTT plasma glucose levels in middle-aged men and women-the Inter99 study. Diabetic Medicine 200926 377-383. (doi:10.1111/j.14645491.2009.02688.x)

98 Faerch K, Vaag A, Holst JJ, Hansen T, Jorgensen T \& BorchJohnsen K. Natural history of insulin sensitivity and insulin secretion in the progression from normal glucose tolerance to impaired fasting glycemia and impaired glucose tolerance: the Inter99 study. Diabetes Care 200932 439-444. (doi:10.2337/dc081195)

99 Unwin N, Shaw J, Zimmet P \& Alberti KG. Impaired glucose tolerance and impaired fasting glycaemia: the current status on definition and intervention. Diabetic Medicine 200219 708-723. (doi:10.1046/j.1464-5491.2002.00835.x)

100 Abdul-Ghani MA, Tripathy D \& DeFronzo RA. Contributions of $\beta$-cell dysfunction and insulin resistance to the pathogenesis of impaired glucose tolerance and impaired fasting glucose. Diabetes Care 200629 1130-1139. (doi:10.2337/dc05-2179)

101 Lokk K, Modhukur V, Rajashekar B, Martens K, Magi R, Kolde R, Koltsina M, Nilsson TK, Vilo J, Salumets A et al. DNA methylome profiling of human tissues identifies global and tissue-specific methylation patterns. Genome Biology 201415 r54. (doi:10.1186/ gb-2014-15-4-r54)

102 Rakyan VK, Down TA, Thorne NP, Flicek P, Kulesha E, Gräf S, Tomazou EM, Bäckdahl L, Johnson N, Herberth M et al. An integrated resource for genome-wide identification and analysis of human tissue-specific differentially methylated regions (tDMRs). Genome Research 200818 1518-1529. (doi:10.1101/ gr.077479.108)

103 Illingworth R, Kerr A, DeSousa D, Jørgensen H, Ellis P, Stalker J, Jackson D, Clee C, Plumb R, Rogers J et al. A novel CpG island set identifies tissue-specific methylation at developmental gene loci. PLoS Biology 20086 e22. (doi:10.1371/journal.pbio.0060022)

104 Jaffe AE \& Irizarry RA. Accounting for cellular heterogeneity is critical in epigenome-wide association studies. Genome Biology 201415 R31. (doi:10.1186/gb-2014-15-2-r31)

105 Chavey A, Ah Kioon MD, Bailbé D, Movassat J \& Portha B. Maternal diabetes, programming of beta-cell disorders and intergenerational risk of type 2 diabetes. Diabetes Metabolism 2014 40 323-330. (doi:10.1016/j.diabet.2014.02.003)

106 Gilbert ER \& Liu D. Epigenetics: the missing link to understanding beta-cell dysfunction in the pathogenesis of type 2 diabetes. Epigenetics 20127 841-852. (doi:10.4161/epi.21238)

107 Su R, Yan J \& Yang H. Transgenerational glucose intolerance of tumor necrosis factor with epigenetic alteration in rat perirenal adipose tissue induced by intrauterine hyperglycemia. Journal of Diabetes Research 20162016 4952801. (doi:10.1155/ 2016/4952801)

108 Volkmar M, Dedeurwaerder S, Cunha DA, Ndlovu MN, Defrance M, Deplus R, Calonne E, Volkmar U, Igoillo-Esteve M, Naamane $\mathrm{N}$ et al. DNA methylation profiling identifies epigenetic dysregulation in pancreatic islets from type 2 diabetic patients. EMBO Journal 201231 1405-1426. (doi:10.1038/emboj.2011.503)

109 Muka T, Nano J, Voortman T, Braun KV, Ligthart S, Stranges S, Bramer WM, Troup J, Chowdhury R, Dehghan A et al. The role of global and regional DNA methylation and histone modifications in glycemic traits and type 2 diabetes: A systematic review. Nutrition, Metabolism and Cardiovascular Diseases 201626 553-566. (doi:10.1016/j.numecd.2016.04.002)

110 Kurdyukov S \& Bullock M. DNA Methylation analysis: choosing the right method. Biology 201653 (doi:10.3390/biology5010003)

111 Chen YA, Lemire M, Choufani S, Butcher DT, Grafodatskaya D, Zanke BW, Gallinger S, Hudson TJ \& Weksberg R. Discovery of cross-reactive probes and polymorphic CpGs in the Illumina Infinium HumanMethylation450 microarray. Epigenetics 20138 203-209. (doi:10.4161/epi.23470)

Received 12 December 2016

Revised version received 16 February 2017

Accepted 22 February 2017 